\title{
EXISTENCE OF UNIQUE SRB-MEASURES IS TYPICAL FOR REAL UNICRITICAL POLYNOMIAL FAMILIES
}

\author{
By HENK BRUIN ${ }^{1}$, WEIXIAO SHEN ${ }^{2}$, SEBASTIAN VAN STRIEN
}

ABSTRACT. - We show that for a one-parameter family of unicritical polynomials $\left\{f_{c}\right\}$ with even critical order $\ell \geqslant 2$, for almost all parameters $c, f_{c}$ admits a unique SRB-measure, being either absolutely continuous, or supported on the postcritical set. As a byproduct we prove that if $f_{c}$ has a Cantor attractor, then it is uniquely ergodic on its postcritical set.

(c) 2006 Published by Elsevier Masson SAS

RÉSUMÉ. - Nous montrons que si $\left\{f_{c}\right\}$ est une famille à un paramètre de polynômes unicritiques dont l'ordre $\ell \geqslant 2$ est pair, alors pour presque toute valeur du paramètre $c, f_{c}$ admet une unique mesure SRB et soit cette mesure est absolument continue, soit son support est l'ensemble postcritique. Nous montrons aussi que, si $f_{c}$ a un attracteur de Cantor, alors $f_{c}$ est uniquement ergodique.

(C) 2006 Published by Elsevier Masson SAS

\section{Introduction and statement of results}

About 10 years ago, Jacob Palis conjectured that "most" dynamical systems have a finite number of metric attractors whose union of basins of attraction has total probability, and that each of these attractors either is a periodic orbit or supports a physical measure, i.e., a measure whose set of typical points has positive Lebesgue measure. The topological version of this conjecture was recently proved in the one-dimensional case: within the space of $C^{\infty}$ one-dimensional maps, hyperbolic maps are dense, see $[19,20]$. This paper deals with 'Lebesgue most' parameters within a family of polynomial maps, and proposes a new strategy for proving a probabilistic version of the above conjecture.

Consider the family $f_{c}(x)=x^{\ell}+c$, where $\ell$ is an even positive integer. Let $\mathcal{M}$ denote the set of parameters $c$ such that $f_{c}$ has a connected Julia set. Then $\mathcal{M} \cap \mathbb{R}$ consists of the parameters $c \in \mathbb{R}$ for which $f_{c}$ has a compact invariant interval, consisting of the (real) points not escaping to infinity. An $f$-invariant measure $\mu$ is called physical or $S R B$ if its basin, i.e., the set $B(\mu)$ of points $x$ such that for all continuous functions $\varphi: \mathbb{R} \rightarrow \mathbb{R}$ one has

$$
\lim _{n \rightarrow \infty} \frac{1}{n} \sum_{k=0}^{n-1} \varphi\left(f^{k}(x)\right)=\int \varphi \mathrm{d} \mu,
$$

\footnotetext{
${ }^{1}$ Supported by EPSRC Grant GR/S91147/01.

${ }^{2}$ Supported by EPSRC Grant GR/R73171/01 and Bai Ren Ji Hua program of the CAS. 
has positive Lebesgue measure. A probability invariant measure which is absolutely continuous w.r.t. the Lebesgue measure is called an acip, and we say that a dynamical system $g: X \rightarrow X$ is uniquely ergodic if there is at most one probability measure on $X$ which is invariant under $g$. There are many parameters $c \in \mathcal{M} \cap \mathbb{R}$ for which $f_{c}$ has no physical measure, or the physical measure is supported on a hyperbolically repelling set, see [16] and also [29]. Our main theorem states that for Lebesgue almost all $c \in \mathcal{M} \cap \mathbb{R}$ there is a unique physical measure.

THEOREM 1. - For Lebesgue-a.e. $c \in \mathcal{M} \cap \mathbb{R}, f_{c}: \mathbb{R} \rightarrow \mathbb{R}$ has a unique physical measure $\mu$. Moreover, either $\mu$ is an acip, or $\mu$ is supported on $\omega(0)$ and $f_{c} \mid \omega(0)$ is uniquely ergodic.

It is well-known, see for example [29, Section III.4], that for all parameters, $f_{c}$ has a unique metric attractor which is one of the following:

1. An attracting periodic orbit.

2. A union of intervals which are cyclically permuted by $f$. This is the finitely renormalizable case.

3. A Cantor set $\omega(0)$ which is equal to $\bigcap_{k} \bigcup_{i=0}^{p_{k}-1} J_{k}$, where $\left\{J_{k}\right\}_{k \in \mathbb{N}}$ is a nested sequence of $p_{k}$-periodic neighborhoods of 0 . This is the infinitely renormalizable case. The basin of $\omega(0)$ has full Lebesgue measure and is of second Baire category.

4. A Cantor set $\omega(0)$, but case 3. does not apply. In this case the basin of $\omega(0)$ has full Lebesgue measure but is of first Baire category. The attractor $\omega(0)$ is called a "wild attractor".

In all of these cases, the basin of the attractor has full Lebesgue measure, and in cases 2-4, Lebesgue measure is ergodic. If in addition, there is an acip $\mu$ (in case 2), then this acip is necessarily the unique SRB-measure, because its set of typical points has full Lebesgue measure. Hence, the basin of the measure $\mu$ from the theorem has full Lebesgue measure.

We should emphasize that if in the above theorem $\operatorname{supp}(\mu)=\omega(0)$, then this need not imply that $\omega(0)$ is the metric attractor. It could, for example, happen that there is a conservative $\sigma$-finite acip $\tilde{\mu}$, such that Lebesgue-a.e. $x$ is typical for both $\mu$ and $\tilde{\mu}$; yet these points visit any set $A$ whose closure is disjoint from $\omega(0)$ with frequency 0 .

For $\ell=2$ a stronger result is known: for almost all $c \in \mathcal{M} \cap \mathbb{R}$, either $f_{c}$ is Collet-Eckmann or $f_{c}$ has a hyperbolic periodic attractor, see $[2,3,22,24]$. However, the geometry of orbits for $\ell=2$ and $\ell>2$ is completely different (for example, wild attractors exist only if $\ell$ is sufficiently large). For this reason several crucial steps of the proofs in those papers fail for the case $\ell>2$. For this reason we use a new approach to this problem in this paper.

Decompose the set $\mathcal{M} \cap \mathbb{R}$ as the union of the following pairwise disjoint sets: $\mathcal{M} \cap \mathbb{R}=$ $\mathcal{A} \cup \mathcal{F} \cup \mathcal{I}$, where

$$
\begin{aligned}
& \mathcal{A}=\left\{c \in \mathcal{M} \cap \mathbb{R} \mid f_{c} \text { has a periodic attractor }\right\}, \\
& \mathcal{F}=\left\{c \in \mathcal{M} \cap \mathbb{R} \backslash \mathcal{A} \mid f_{c} \text { is at most finitely renormalizable }\right\}, \\
& \mathcal{I}=\left\{c \in \mathcal{M} \cap \mathbb{R} \mid f_{c} \text { is infinitely renormalizable }\right\} .
\end{aligned}
$$

In the first case, $f_{c}$ has an SRB-measure supported on the periodic attractor. In the third case, $\omega(0)$ is a Cantor set and carries a unique invariant probability measure, which is also the unique SRBmeasure. In fact, $(\omega(0), f)$ is isomorphic to an adding machine. See [29, Section II.5] and [15, Chapter 11] for proofs of these statements. So to prove Theorem 1, we only need to consider the second case.

Let us further decompose the space of finitely renormalizable maps $\mathcal{F}$ as $\mathcal{F}=\bigcup_{n=0}^{\infty} \mathcal{F}^{n}$, where $\mathcal{F}^{n}$ denotes the subset of $\mathcal{F}$ consisting of parameters $c$ for which $f_{c}$ is exactly $n$ times renormalizable. Most of our effort will be put into the case $c \in \mathcal{F}^{0}$ as the finitely renormalizable case can be reduced by a suitable return map to the non-renormalizable case. Let us use $\mathcal{F}_{r}^{0}$ to

$4^{\mathrm{e}}$ SÉRIE - TOME $39-2006-\mathrm{N}^{\circ} 3$ 
denote the subset of $\mathcal{F}^{0}$ consisting of parameters $c$ for which $f_{c}$ has a recurrent critical point. By a classical result of Misiurewicz, $f_{c}$ has an acip for any $c \in \mathcal{F}^{0} \backslash \mathcal{F}_{r}^{0}$, see for example [29].

The case when $f_{c}$ has a recurrent critical point is much more tricky. To explain our strategy, we need some more terminology. Let us say that an open interval $I$ is nice if $f^{n}(\partial I) \cap I=\emptyset$ for all $n \geqslant 0$. An interval $J \ni 0$ is called a child of $I$ if it is a unimodal pullback of $I$, i.e., if there exist an interval $\tilde{J}$ containing the critical value $c$ and an integer $s \geqslant 1$ so that $f^{s-1}: \tilde{J} \rightarrow I$ is a homeomorphism and $J=f^{-1}(\tilde{J})$. If $c \in \mathcal{F}_{r}^{0}$ and there exists a nice interval $I \ni 0$ with infinitely many children, then we say that $f_{c}$ is reluctantly recurrent; otherwise it is called persistently recurrent. Let us say that a parameter $c \in \mathcal{F}_{r}^{0}$ has decaying geometry property if either

- $f_{c}$ is reluctantly recurrent, or

- $f_{c}$ is persistently recurrent and there exists a sequence of nice intervals $\Gamma^{0} \supset \Gamma^{1} \supset \cdots \ni 0$ such that for each $n \geqslant 0, \Gamma^{n+1}$ is the smallest child of $\Gamma^{n}$, and so that $\left|\Gamma^{n+1}\right| /\left|\Gamma^{n}\right| \rightarrow 0$ as $n \rightarrow \infty$.

Let $\mathcal{D G}$ denote the collection of parameters $c$ for which $f_{c}$ satisfies the decaying geometry condition. We should note that if $\ell=2, \mathcal{F}_{r}^{0} \subset \mathcal{D G}$ (and in fact, the decay is at least exponentially fast, see $[14,21,35])$. We will first deal with the parameters $c \in \mathcal{F}_{r}^{0} \backslash \mathcal{D G}$, and show that $f_{c} \mid \omega(0)$ is uniquely ergodic, and there is a unique SRB-measure which is either an acip or the invariant probability measure supported on $\omega(0)$. In Section 2 we study the combinatorics of $\omega(0)$ when $f_{c}$ is persistently recurrent, and we introduce a notion of combinatorial complexity, see Definition 1. Based on this notion we state the following

THEOREM 2. - If a unimodal map $f$ is persistently recurrent and has low combinatorial complexity, then $f \mid \omega(0)$ is uniquely ergodic. Moreover, if $f$ is $C^{3}$, then there is a unique $S R B$-measure which is either an acip, or is the unique invariant probability measure supported on $\omega(0)$.

It follows from Lebesgue ergodicity of unimodal maps without periodic attractors that an acip is indeed the unique SRB-measure. The link with the decaying geometry property is made in the following:

THEOREM 3. - If $c \in \mathcal{F}_{r}^{0} \backslash \mathcal{D G}$, then $f_{c}$ has low combinatorial complexity (and hence Theorem 2 applies).

To deal with the set $\mathcal{D G}$, we shall carry out a parameter exclusion argument in spite of the fact that $\left|\Gamma^{n+1}\right| /\left|\Gamma^{n}\right|$ need not decay exponentially.

For a subset $A$ of a bounded interval $I$, and $\gamma \geqslant 1$,

$$
\operatorname{Cap}_{\gamma}(A, I)=\sup _{h} \frac{|h(A)|}{|h(I)|},
$$

where $h$ runs over all $\gamma$-quasisymmetric maps from $I$ into $\mathbb{R}$. Moreover, let $\mathcal{S C}$ be the subset of $\mathcal{F}_{r}^{0}$ consisting of all the parameters $c$ such that for any $\alpha>0$ the following summability condition holds:

$$
\sum_{n=0}^{\infty} \frac{1}{\left|D f_{c}^{n}(c)\right|^{\alpha}}<\infty \quad(\mathcal{S C}) .
$$

By [31], for any $c \in \mathcal{S C}, f_{c}$ has an acip, and the remarks below Theorem 1 show that this acip is the unique SRB-measure. (In fact, by [8], this acip has decay of correlations faster than any polynomial rate.)

THEOREM 4. - The set $\mathcal{S C}$ has full Lebesgue measure in $\mathcal{F}_{r}^{0} \cap \mathcal{D G}$. To describe the geometry of the set $\mathcal{S C}$ more precisely, for every $c \in \mathcal{D G}$ and every $\varepsilon>0$ and $\gamma>1$, there exists 
a neighborhood $J \ni c$, such that

$$
\operatorname{Cap}_{\gamma}((J \backslash \mathcal{S C}), J)<\varepsilon .
$$

To prove this theorem we shall follow the idea of $[3,22]$, which uses complex method in an essential way. The new ingredient here is a different strategy to obtain dilatation control of "pseudo-conjugacies". In quadratic case, such control was deduced from "linear growth of the principal moduli" which does not hold in our case (even for maps satisfying our decaying geometry condition). Instead, we shall prove in the case $c \in \mathcal{D G}$, that there exists a sequence of critical puzzle pieces for which the relative size of the first return domains is arbitrarily 'small', see Theorem 6 . This result implies dilatation control for the pseudo-conjugacies by an argument used previously in $[17,32,36]$.

Before giving a formal proof of Theorem 1, let us summarize the strategy schematically in the following way:

$$
\begin{aligned}
& c \in \mathcal{F}_{r}^{0} \backslash \mathcal{D} \mathcal{G}^{\text {Theorem }}{ }^{3} \text { low combin. complexity } \stackrel{\text { Theorem } 2}{\Longrightarrow} \text { unique ergodicity; } \\
& \text { a.e. } c \in \mathcal{F}_{r}^{0} \cap \mathcal{D G} \stackrel{\text { Theorem } 4}{\Longrightarrow} c \in \mathcal{S C} \stackrel{\text { by [31] }}{\Longrightarrow} \text { has an SRB; } \\
& c \in \mathcal{F}^{0} \backslash \mathcal{F}_{r}^{0} \Longrightarrow c \text { is Misiurewicz } \Longrightarrow \text { has an SRB; }
\end{aligned}
$$

while for renormalizable maps, the same argument goes through by considering a renormalization.

Proof of Theorem 1. - Let Good denote the set of all parameters $c \in \mathcal{M} \cap \mathbb{R}$ for which $f_{c}$ has a unique SRB measure and let $B a d=(\mathcal{M} \cap \mathbb{R}) \backslash$ Good. We need to show that $B a d$ has Lebesgue measure zero. As we have mentioned before, $B a d \subset \mathcal{F}$. So it suffices to show that for each $n \geqslant 0$, the set $\operatorname{Bad}_{n}:=B a d \cap \mathcal{F}^{n}$ has no Lebesgue density point. In the case $n=0$, this follows from Theorems 2, 3 and 4 .

Let us now fix $n$ and $c \in B a d_{n}$. Then $f$ has a non-periodic recurrent critical point, and there exists a neighborhood $K \ni c$ such that for each $c^{\prime} \in K, f_{c^{\prime}}$ is at least $n$ times renormalizable. For each $c^{\prime}$ let $\chi\left(c^{\prime}\right)$ denote the parameter in $\mathcal{M} \cap \mathbb{R}$ such that $f_{\chi\left(c^{\prime}\right)}$ is hybrid conjugate to the $n$-th renormalization of $f_{c^{\prime}}$. By the qc-Theorem in [23] (qc will always stand for quasiconformal), the map $\chi: K \rightarrow \chi(K)$ is $\gamma$-quasisymmetric for some $\gamma>1$. Note that for any $c^{\prime} \in K, c^{\prime} \in B a d_{n}$ implies that $\chi\left(c^{\prime}\right) \in B a d_{0}$. In particular, $f_{\chi(c)}$ satisfies the decay of geometry condition, so we may apply Theorem 4 to obtain a sequence $J_{n}$ of neighborhoods of $\chi(c)$, such that $\left|J_{n}\right| \rightarrow 0$ and $\operatorname{Cap}_{\gamma}\left(\left(J_{n} \backslash \mathcal{S C}\right) \mid J_{n}\right) \rightarrow 0$, as $n \rightarrow \infty$. It follows that $\left|\chi^{-1}\left(J_{n}\right) \cap \operatorname{Bad}_{n}\right| /\left|\chi^{-1}\left(J_{n}\right)\right| \rightarrow 0$ as $n \rightarrow \infty$, i.e., $c$ is not a Lebesgue density point of $B a d_{n}$. This completes the proof.

Finally, we shall show in Section 3 that the decaying geometry condition excludes existence of Cantor attractors. Therefore we have

THEOREM 5. - If $f$ has a Cantor attractor $\omega(0)$ (of solenoid type, or a "wild attractor"), then $f$ has low combinatorial complexity and hence $f \mid \omega(0)$ is uniquely ergodic.

\subsection{Organization of the paper and some comments on the proof}

Section 2 is devoted to proving Theorem 2 and we start by showing that if $f$ is persistently recurrent and one has low combinatorial complexity, then $f \mid \omega(0)$ is uniquely ergodic, see Proposition 1. This is done by showing that certain transition matrices act as contractions in the projective Hilbert metric. In Section 3 we use real bounds to complete the proof of Theorem 3. The proof of Theorem 5 is also given in that section. The remainder of the paper is devoted to the

$4^{e}$ SÉRIE - TOME $39-2006-\mathrm{N}^{\circ} 3$ 
proof of Theorem 4. In Section 4 we review how the combinatorics of Yoccoz puzzles changes with the parameter. In Section 5, we study the geometry of the Yoccoz puzzle for maps $f_{c}$ with decaying geometry property, and state and prove Theorem 6 . In Section 6, we convert this result to an estimate of the dilatation of pseudo-conjugacies. Such an estimate will be crucial when we go from dynamical to parameter space in the proof of Theorem 4 in Section 7.

Most of the proofs in this paper can be extended to multimodal families. However, in order to go from dynamical space to parameter space we use Proposition 7, which essentially follows from Lemma 9. In higher dimensions, Lemma 9 is no longer true. For example, let $\mathcal{H}$ be the space of biholomorphic maps $\varphi: \mathbb{C} \times \mathbb{C} \rightarrow \mathbb{C} \times \mathbb{C}$ such that $\varphi(\mathbb{R} \times \mathbb{R}) \subset \mathbb{R} \times \mathbb{R}$. Let $\lambda$ be the Lebesgue measure on $\mathbb{R} \times \mathbb{R}$ and define

$$
A(\varphi)=\frac{\lambda(\varphi((-\varepsilon, \varepsilon) \times(-\varepsilon, \varepsilon)))}{\lambda(\varphi((-1,1) \times(-1,1)))},
$$

then $\sup _{\varphi \in \mathcal{H}} A(\varphi)$ need not be small if $\varepsilon$ is small. So even if the holomorphic motion in Lemma 9 is trivial $(h=\mathrm{id})$ going from dynamical to parameter space is more difficult.

Throughout the paper we shall say that $A \Subset B$ if the closure of $A$ is contained in the interior of $B$. For any interval $I$, let $\alpha I$ denote the interval of length $\alpha|I|$ that is concentric with $I$.

\section{A condition for unique ergodicity in the persistently recurrent case}

In this section, let $f$ be an arbitrary $C^{2}$ unimodal map with a non-flat critical point $c_{0}=0$. We shall assume that the critical point is recurrent, but not periodic. The goal is to give a sufficient condition for $f \mid \omega(0)$ to be uniquely ergodic. So we shall assume that $f$ is not renormalizable; if $f$ is finitely renormalizable we pass to the "deepest" renormalization, whereas for infinitely renormalizable maps, $\omega(0)$ is an attractor and $f \mid \omega(0)$ is isomorphic to the adding machine (defined by "adding 1 and carry") on the space $\left\{\left(x_{i}\right)_{i=1}^{\infty} \mid x_{1} \in\left\{0, \ldots, p_{1}-1\right\}\right.$, $x_{i} \in\left\{0, \ldots, \frac{p_{i}}{p_{i-1}}-1\right\}$ for $\left.i \geqslant 2\right\}$. Here $p_{i}$ is the period of the $i$-th periodic interval. Such adding machines are well-known to be uniquely ergodic, see [15].

\subsection{Construction of the nest of children}

Recall that an open interval $\Gamma$ is called nice if $f^{n}(\partial \Gamma) \cap \Gamma=\emptyset$ for all $n \geqslant 1$. For any nice interval $\Gamma \ni 0$, let $R_{\Gamma}: \Gamma \rightarrow \Gamma$ be the first return map; it has one central unimodal branch (which contains the critical point 0 ) and in general infinitely many non-central branches. Let $\rho(\Gamma)$ be the collection of return domains of $\Gamma$ that intersect $\omega(0)$. A child $\Gamma^{\prime}$ of $\Gamma$ is a neighborhood of 0 such that there exists a neighborhood $\widetilde{\Gamma}$ of $c_{1}:=f(0)$ such that $f^{-1}(\widetilde{\Gamma})=\Gamma^{\prime}$ and $f^{s-1}: \widetilde{\Gamma} \rightarrow \Gamma$ is monotone onto for some $s \geqslant 1$. The children of $\Gamma$ are again nice, nested neighborhoods of 0 . Each nice neighborhood has at least one, and if $f$ is not renormalizable at least two children.

If $f$ is persistently recurrent then (by definition) each nice neighborhood $\Gamma$ of 0 has only finitely many children (cf. [7,39]). Note that persistent recurrence of $f$ implies that $\omega(0)$ is a minimal Cantor set, see [21]. Making this assumption, let $\Gamma^{1}$ be the smallest child of $\Gamma^{0}$. Continue by induction, $\Gamma^{n+1}$ being the smallest child of $\Gamma^{n}$. Let $s_{n}$ be the iterate such that $f^{s_{n}-1}$ maps a neighborhood $\widetilde{\Gamma}^{n+1}$ of $f\left(\Gamma^{n+1}\right)$ monotonically onto $\Gamma^{n}$.

LEMMA 1. - If $\Gamma^{n+1}$ is the smallest child of $\Gamma^{n}$, then for each $J \in \rho\left(\Gamma^{n}\right)$, there exists an integer $0 \leqslant t<s_{n}$ such that $f^{t}\left(\Gamma^{n+1}\right) \subset J$. In particular, the existence of a smallest child implies that $\# \rho\left(\Gamma^{n}\right)<\infty$. 
Proof. - If $J$ is the central domain, then $t=0$ works. Take $J \in \rho\left(\Gamma^{n}\right)$ non-central, and let $t^{\prime}$ be minimal such that $f^{t^{\prime}}(0) \in J$. Then there exists a neighborhood $U$ of $c_{1}$ such that $f^{t^{\prime}-1}: U \rightarrow J$ is monotone onto, and iterating some $\beta=\beta(J)$ steps more, $U$ is mapped monotonically onto $\Gamma^{n}$. Therefore $f^{-1}(U)$ is a child of $\Gamma^{n}$. If $t^{\prime} \geqslant s_{n}$, then this child is actually smaller than $\Gamma^{n+1}$, a contradiction.

\subsection{Unique ergodicity}

Let $I^{0}:=\Gamma^{n}$ be any interval in the chain of smallest children. Let $I^{1}:=\mathcal{L}_{0}\left(I^{0}\right)$ be the central return domain of $R_{\Gamma^{n}}=: R_{0}$. This domain is again nice, so it has a central return domain $I^{2}$ under the return map $R_{1}:=R_{I^{1}}: I^{1} \rightarrow I^{1}$. Continue by induction to construct the principal nest of $\Gamma^{n}$ by defining $I^{i+1}$ as the central return domain of the return map $R_{i}$ to the previous central domain $I^{i}$. Then for some $r, I^{r} \supsetneq \Gamma^{n+1} \supset I^{r+1}$. For any $y \in \Gamma^{n} \cap \omega(0)$, the first landing map of $y$ to $\Gamma^{n+1}$ can be decomposed into return maps $R_{i}$. Indeed, write

$$
R(z)= \begin{cases}R_{0}(z) & \text { if } z \in I^{0} \backslash I^{1} \\ R_{i-1}(z) & \text { if } z \in I^{i} \backslash I^{i+1} \text { and } i \geqslant 1 .\end{cases}
$$

Let $k=k(y) \geqslant 0$ be such that $R^{k}(y)$ is the first landing of $y$ into $\Gamma^{n+1}$, and for $0 \leqslant l \leqslant k$, write $\alpha_{l}(y)=i$ if $R^{l}(y) \in I^{i} \backslash I^{i+1}$. Define the combinatorial complexity of $y \in \Gamma^{n}$ to be

$$
\mathcal{G}_{n}(y)=\#\left\{0 \leqslant l<k \mid \alpha_{l}(y) \leqslant \alpha_{l+1}(y)\right\} .
$$

Note that $\mathcal{G}_{n}(y) \geqslant 1$, unless $y \in I^{r}$. As $\omega(0)$ is a minimal Cantor set, $k(y)$ is uniformly bounded for $y \in \omega(0) \cap \Gamma^{n}$. In particular, we have

$$
\mathcal{G}_{n}:=\sup _{y \in \omega(0) \cap \Gamma^{n}} \mathcal{G}_{n}(y)<\infty .
$$

Note that if $f$ is not renormalizable, then $\mathcal{G}_{n} \geqslant 1$ for all $n$.

DEFINITION 1.- If $f$ is persistently recurrent, then we say that it has low combinatorial complexity if $\sum_{n \geqslant 0} \frac{1}{\mathcal{G}_{n}}=\infty$.

PROPOSITION 1 (Non-unique ergodicity implies growing combinatorial complexity). - Let $f$ be a persistently recurrent non-flat $C^{2}$ unimodal map with low combinatorial complexity, then $f \mid \omega(0)$ is uniquely ergodic.

Proof. - For each $n$, the return domains $\rho_{n}:=\rho\left(\Gamma^{n}\right) \operatorname{cover} \omega(0) \cap \Gamma^{n}$. For each $J \in \rho$, let $\tau_{J}$ be the return time of $J$ into $\Gamma^{n}$. So if $J$ is non-central, then $f^{\tau_{J}}: J \rightarrow \Gamma^{n}$ is monotone onto, and if $J:=\mathcal{L}_{0}\left(\Gamma^{n}\right)$ is the central return domain, then there exists an interval $\widetilde{\mathcal{L}}_{0}\left(\Gamma^{n}\right) \supset f\left(\mathcal{L}_{0}\left(\Gamma^{n}\right)\right)$ such that $f^{\tau_{J}-1}: \widetilde{\mathcal{L}}_{0}\left(\Gamma^{n}\right) \rightarrow \Gamma^{n}$ is monotone onto. Let $H_{n}$ be the collection of intervals of the following form: (i) $f^{k}(J)$ for $J \in \rho_{n}$ non-central and $0 \leqslant k<\tau_{J}$, (ii) the central return domain $\mathcal{L}_{0}\left(\Gamma^{n}\right)$ and (iii) $f^{k-1}\left(\widetilde{\mathcal{L}}_{0}\left(\Gamma^{n}\right)\right)$ for $0<k<\tau_{\mathcal{L}_{0}\left(\Gamma^{n}\right)}$. Then $H_{n}$ is a cover of $\omega(0)$. Since $f$ is assumed to be $C^{2}$ and have non-flat critical point, it has no wandering intervals (see [29]) so that $\sup \left\{\operatorname{diam}(J) \mid J \in H_{n}\right\} \rightarrow 0$ as $n \rightarrow \infty$. Therefore $\bigvee_{n}\left(H_{n} \cap \omega(0)\right)$ generates the Borel $\sigma$-algebra on $\omega(0)$.

The idea of the proof is now to show that for each $J \in H_{n}, n \in \mathbb{N}$, and $y \in \omega(0)$, the visit frequency $\lim _{m \rightarrow \infty} \frac{1}{m}\left\{0 \leqslant i<m \mid f^{i}(y) \in J\right\}$ exists and is independent of $y$. For any $J^{\prime} \in H_{n}$ and $J \in \rho_{n}$, there is at most one $k<\tau_{J}$ such that $f^{k}(J) \cap J^{\prime} \neq \emptyset$ and for this $k, f^{k}(J) \subset J^{\prime}$. This implies that the visit frequency to $J^{\prime} \in H_{n}$ is the sum of the visit frequencies of all $J \in \rho_{n}$

$4^{\text {e }}$ SÉRIE - TOME $39-2006-\mathrm{N}^{\circ} 3$ 
that visit it in iterates $0, \ldots, \tau_{J}-1$. It therefore suffices to compute visit frequencies to $J \in \rho_{n}$. A priori, these limits need not exist, so we replace them by visit frequency set $\gamma_{n}$ consisting of all visit frequency vectors $\tilde{\gamma}_{n}$ obtained from some $y \in \omega(c)$ and subsequence $\left\{m_{k}\right\}$. In other words, $\tilde{\gamma}_{n} \in \gamma_{n}$ if there is a subsequence $\left\{m_{k}\right\}$ and $y \in \omega(0)$ such that the components

$$
\tilde{\gamma}_{n}(J)=\lim _{k \rightarrow \infty} \frac{1}{m_{k}} \#\left\{0 \leqslant i<m_{k} \mid f^{i}(y) \in J\right\}, \quad J \in \rho_{n}
$$

exist. Note that $\sum_{J \in \rho_{n}} \tilde{\gamma}_{n}(J)=1$ for all $\tilde{\gamma}_{n} \in \gamma_{n}$.

The next step is to express $\gamma_{n}(J)$ as an set-valued linear combination of $\left\{\gamma_{n+1}\left(J^{\prime}\right) \mid\right.$ $\left.J^{\prime} \in \rho_{n+1}\right\}$. The corresponding linear transformation $A_{n}: \mathcal{C}_{n+1} \rightarrow \mathcal{C}_{n}$, for cones $\mathcal{C}_{n}:=\mathbb{R}_{+}^{\# \rho_{n}}$ (where $\mathbb{R}_{+}=[0, \infty)$ ), is determined by the combinatorics of the return map $R_{n}$ to $\Gamma^{n}$. Using the projective Hilbert metric $\Theta$, we will prove that infinite compositions of the transformations $A_{n}$ contract the cones to halflines $\ell_{n} \subset \mathcal{C}_{n}$. Intersecting $\ell_{n}$ with the unit simplex in $\mathcal{C}_{n}$ gives a single point whose coordinates express the visit frequencies to the elements $J \in \rho_{n}$, and these frequencies exist as limits and are independent of $y \in \omega(0)$.

Let us study these linear transformations $A_{n}$ in more detail. Let $a_{J, \tilde{J}}$ indicate the number of visits of $\tilde{J} \in \rho_{n+1}$ to $J \in \rho_{n}$ in the iterates $0, \ldots, \tau_{\tilde{J}}-1$. Then $\left(a_{J, \tilde{J}}\right)_{J \in \rho_{n}, \tilde{J} \in \rho_{n+1}}$ is an $\# \rho_{n} \times \# \rho_{n+1}$ matrix which we will identify with the transformation $A_{n}$. Then, as a set-valued matrix product,

$$
\gamma_{n}(J) \subset \frac{1}{N_{n}} \sum_{\tilde{J} \in \rho_{n+1}} a_{J, \tilde{J}} \gamma_{n+1}(\tilde{J}):=\left\{\frac{1}{N_{n}} \sum_{\tilde{J} \in \rho_{n+1}} a_{J, \tilde{J}} z \mid z \in \gamma_{n+1}(\tilde{J})\right\}
$$

for some normalizing constant $N_{n}$. Let $\gamma_{n}$ be the set of $\# \rho_{n}$-tuples of visit frequencies $\left\{\tilde{\gamma}_{n}(J) \mid J \in \rho_{n}\right\}$ written as a vertical column. Then composing matrices $A_{n}$, we find

$$
\gamma_{n}=\frac{1}{N_{n, m}} A_{n} \cdot A_{n+1} \cdots A_{m-1} \gamma_{m} .
$$

Disregarding the normalizing constants $N_{n, m}$, we find that $\gamma_{n}$ is a single vector, i.e., independent of $y$, if and only if

$$
\ell_{n}:=\bigcap_{m>n} A_{n} \cdot A_{n+1} \cdots A_{m-1}\left(\mathcal{C}_{m}\right)
$$

is a half-line, and in that case $\gamma_{n}$ is the intersection of $\ell_{n}$ and the unit simplex $\left\{x \in \mathcal{C}_{n} \mid\right.$ $x_{i} \geqslant 0$ and $\left.\sum_{i=1}^{\# \rho_{n}} x_{i}=1\right\}$. As a result, the visit frequency to any $J \in \rho_{n}$ and any $n \geqslant 0$ is determined independently of $y \in \omega(0)$, and consequently, the visit frequencies to $J^{\prime} \in H_{n}$ exist independently of $y$. By Kolmogorov's extension theorem, this uniquely determines the invariant measure $\mu$.

Note that the entries $a_{J, \tilde{J}}$ of $A_{n}$ are strictly positive. This is a consequence of Lemma 1, and it is here that we effectively use the fact that $\Gamma^{n+1}$ is the smallest child of $\Gamma^{n}$. More precisely, if the matrix $A_{n}^{+}$records all the visits of $\tilde{J}$ 's in $\rho_{n+1}$ to $J$ 's in $\rho_{n}$ before iterate $s_{n}$, then $A_{n}^{+}$ is already strictly positive. Moreover, for each $t<s_{n}, f^{t}\left(\Gamma^{n+1}\right)$ intersects at most one return domain $J \in \rho_{n}$. Thus all columns of $A_{n}^{+}$are identical. The differences of visits of the respective $\tilde{J}$ 's occur only after the iterate $s_{n}$, and are recorded in the matrix $A_{n}^{-}:=A_{n}-A_{n}^{+}$. Let us write $a_{J, \tilde{J}}^{+}$and $a_{J, \tilde{J}}^{-}$for the entries of $A_{n}^{+}$and $A_{n}^{-}$respectively. 
LEMMA 2. - For each $n$ and $J \in \rho_{n}, \tilde{J} \in \rho_{n+1}$ we have $a_{J, \tilde{J}}^{-} \leqslant 2 \mathcal{G}_{n} a_{J, \tilde{J}}^{+}$, where $\mathcal{G}_{n}$ denotes the combinatorial complexity as in Eq. (3).

Proof. - Given $x \in \omega(0) \cap \Gamma^{n+1}$, write $y_{0}=y=f^{s_{n}}(x)$ and $y_{l}=R^{l}(y)$, where $R$ is as in Eq. (2). Abbreviate $\alpha_{l}=\alpha_{l}(y)$. For $i \geqslant 1, R\left|\left(I^{i} \backslash I^{i-1}\right)=R_{i-1}\right|\left(I^{i} \backslash I^{i-1}\right)$ is the central branch of the return map to $I^{i-1}$; let $t_{i}$ be such that $R \mid I^{i}=f^{t_{i}}$.

CLAIM 1. - If $\alpha_{l}=0$, i.e., $y_{l}$ belongs to a non-central domain $J \in \rho\left(I^{0}\right)$, and $R \mid J=f^{t}$, then $t \leqslant s_{n}$. Moreover, $1=\#\left\{0 \leqslant i<t \mid f^{i}\left(y_{l}\right) \in J\right\} \leqslant \#\left\{0 \leqslant i<s_{n} \mid f^{i}(0) \in J\right\}$.

Proof. - By Lemma 1, there exists $t^{\prime}>0$ such that $f^{t^{\prime}}(0) \in J$, and hence $f^{s_{n}-t^{\prime}}(J) \cap I^{0} \neq \emptyset$. Therefore $t \leqslant s_{n}-t^{\prime}<s_{n}$. The second statement of this claim follows because $R \mid J$ is the first return map to $I^{0}$.

CLAIM 2. - Assume that there exist $l<l^{\prime}$ such that

$$
\alpha_{l}>\alpha_{l+1}>\cdots>\alpha_{l^{\prime}}
$$

then $R^{l^{\prime}-l}\left(y_{l}\right)=f^{t}\left(y_{l}\right)$ for some $t \leqslant s_{n}$. A fortiori, $\#\left\{0 \leqslant i<t \mid f^{i}\left(y_{l}\right) \in J\right\} \leqslant \#\left\{0 \leqslant i<s_{n} \mid\right.$ $\left.f^{i}(0) \in J\right\}$ for each $J \in \rho\left(I^{0}\right)$.

Proof. - Since $f$ is not renormalizable, there exists at least one non-central return domain $J$ of $I^{0}$. Therefore there exists a maximal $s_{n}^{\prime}<s_{n}$ and $J$ such that $f^{s_{n}^{\prime}-1}\left(\widetilde{\Gamma}^{n+1}\right)=J$, where $\widetilde{\Gamma}^{n+1}$ is the one-sided neighborhood of $f\left(\Gamma^{n+1}\right)$ that maps onto $I^{0}$ under $f^{s_{n}}$. Since $I^{\alpha_{l}} \supset \Gamma^{n+1}$, $f^{s_{n}^{\prime}}\left(I^{\alpha_{l}}\right)$ contains at least one boundary point of $J$. But the forward orbit of $\partial J$ is disjoint from the open interval $I^{0}$, and therefore the return time $t_{\alpha_{l}} \leqslant s_{n}^{\prime}$. Furthermore, if $f^{j}\left(y_{l}\right) \in J$ for some $J \in \rho\left(I^{0}\right)$ and $j<t_{\alpha_{l}}$ while $f^{j}\left(\Gamma^{n+1}\right) \not \subset J$, then $f^{j}\left(I^{\alpha_{l}}\right)$ contains a boundary point of $J$. This would contradict that $f^{t_{\alpha_{l}}}\left(I^{\alpha_{l}}\right) \subset I^{\alpha_{l}-1}$. Therefore $y_{l}$ and $\Gamma^{n+1}$ visit the same return domains along the iterates $0 \leqslant j<t_{\alpha_{l}}$. This proves Claim 2 when $l^{\prime}=l+1$.

If $l^{\prime}>l+1$, then $f^{t_{\alpha_{l}}}\left(\Gamma^{n+1}\right) \subset f^{t_{\alpha_{l}}}\left(I^{\alpha_{l}}\right) \subset I^{\alpha_{l+1}}$. Hence we can repeat the argument for the iterates $t_{\alpha_{l}} \leqslant j<t_{\alpha_{l}}+t_{\alpha_{l+1}}$, etc.

In fact, the same argument also proves:

Claim 3. - Assume that $l$ is such that $0<\alpha_{l} \leqslant \alpha_{l+1}$. Then $R\left(y_{l}\right)=f^{t}$ for some $t \leqslant s_{n}$ and $\#\left\{0 \leqslant i<t \mid f^{i}\left(y_{l}\right) \in J\right\} \leqslant \#\left\{0 \leqslant i<s_{n} \mid f^{i}(0) \in J\right\}$ for each $J \in \rho\left(I^{0}\right)$.

To prove the lemma, take any $x \in \tilde{J} \in \rho\left(\Gamma^{n+1}\right)$, and decompose $\{0, \ldots, k\}$ into strings $l, l+1, \ldots, l^{\prime}$ that satisfy the hypotheses of one of the thee above claims. If $\alpha_{l} \leqslant \alpha_{l+1}$, then Claim 1 or 3 holds for $l$, whereas for any maximal string $\alpha_{l}>\alpha_{l+1}>\cdots>\alpha_{l^{\prime}}$, Claim 1 or 3 holds for $l^{\prime}$. By definition of $\mathcal{G}_{n}$, there are at most $2 \mathcal{G}_{n}$ such strings, and each such strings, $\#\left\{0 \leqslant i<t \mid f^{i}\left(y_{l}\right) \in J\right\} \leqslant \#\left\{0 \leqslant i<s_{n} \mid f^{i}(0) \in J\right\}$ for each $J \in \rho\left(\Gamma^{n}\right)$. Hence $a_{J, \tilde{J}}^{+} \leqslant 2 \mathcal{G}_{n} a_{J, \tilde{J}}^{-}$as asserted.

To conclude the proof of Proposition 1, we will show that the matrices $A_{n}$ act as contractions in the projective Hilbert metric. Given $v, w \in \mathcal{C}_{n+1}$, define this pseudo-metric as

$$
\Theta_{n+1}(v, w)=\log \left(\frac{\inf \left\{\mu \mid \mu v-w \in \mathcal{C}_{n+1}\right\}}{\sup \left\{\lambda \mid w-\lambda v \in \mathcal{C}_{n+1}\right\}}\right) .
$$

Let $A_{n}: \mathcal{C}_{n+1} \rightarrow \mathcal{C}_{n}$ be a linear map. It is shown in e.g. [4] that $\Theta_{n}\left(A_{n} v, A_{n} w\right) \leqslant$ $\tanh (D / 4) \Theta_{n+1}(v, w)$ for $D=\sup _{v^{\prime}, w^{\prime} \in \mathcal{C}_{n+1}} \Theta_{n}\left(A_{n} v^{\prime}, A_{n} w^{\prime}\right)$. In particular, $A_{n}$ is a contraction if $A_{n}$ maps $\partial \mathcal{C}_{n+1} \backslash\{0\}$ into the interior of $\mathcal{C}_{n}$. By strict positivity of the $A_{n}$, this is true

$4^{\text {e }}$ SÉRIE - TOME $39-2006-\mathrm{N}^{\circ} 3$ 


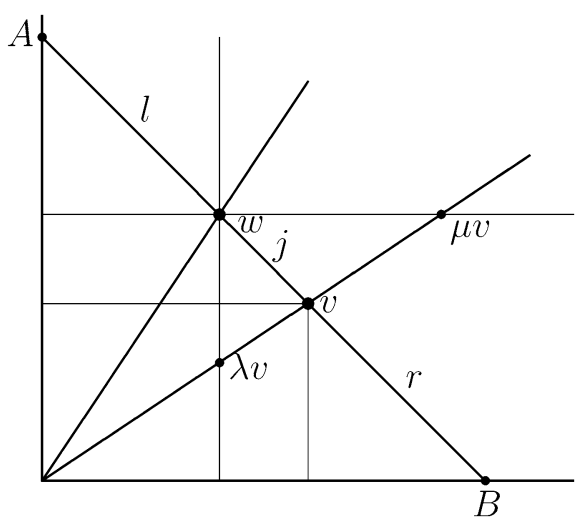

Fig. 1. Illustration of the Hilbert metric.

for all $n$. By Lemma 2, each element in $A_{n}^{-}$is at most $2 \mathcal{G}_{n}$ times the corresponding element in $A_{n}^{+}$. Therefore, when comparing two elements $a_{J, J^{\prime}}$ and $b_{J, J^{\prime \prime}}$ in the same $J$-row of $A_{n}$, we always find that $\frac{1}{1+2 \mathcal{G}_{n}} a_{J, J^{\prime}} \leqslant b_{J, J^{\prime \prime}} \leqslant\left(1+2 \mathcal{G}_{n}\right) a_{J, J^{\prime}}$. Therefore, if $\mu, \lambda>0$ are such that $\mu v-w \notin \mathcal{C}_{n+1}$ and $w-\lambda v \notin \mathcal{C}_{n+1}$, then $\frac{\mu}{\lambda} \leqslant\left(1+2 \mathcal{G}_{n}\right)^{2}$. The contraction factor becomes

$$
\begin{aligned}
\tanh (D / 4) & \leqslant \frac{e^{\frac{1}{2} \log \left(1+2 \mathcal{G}_{n}\right)}-e^{-\frac{1}{2} \log \left(1+2 \mathcal{G}_{n}\right)}}{e^{\frac{1}{2} \log \left(1+2 \mathcal{G}_{n}\right)}+e^{-\frac{1}{2} \log \left(1+2 \mathcal{G}_{n}\right)}} \\
& =\frac{\sqrt{1+2 \mathcal{G}_{n}}-\sqrt{\frac{1}{1+2 \mathcal{G}_{n}}}}{\sqrt{1+2 \mathcal{G}_{n}}+\sqrt{\frac{1}{1+2 \mathcal{G}_{n}}}} \\
& =1-\frac{2}{\left(1+2 \mathcal{G}_{n}\right)\left(1+\frac{1}{1+2 \mathcal{G}_{n}}\right)} \leqslant 1-\frac{1}{1+2 \mathcal{G}_{n}} .
\end{aligned}
$$

Therefore $\ell_{n}$ is indeed a line if $\prod_{m \geqslant n}\left(1-\frac{1}{1+2 \mathcal{G}_{m}}\right)=0$, which is equivalent to $\sum_{n \geqslant 0} \frac{1}{\mathcal{G}_{n}}=\infty$.

Remark 1. - A different way of regarding the Hilbert metric is the following, see Fig. 1. The vectors $v$ and $w$ span a plane $V$, which contains the line connecting $v$ and $w$. Let $A$ and $B$ be the intersections of this line with those coordinate hyperplanes that $V$ intersects ( $A$ or $B$ could be $\infty$ ). The points $v, w, A$ and $B$ bound an arc and divide it into three pieces; call the middle piece $j$ and the other pieces $l$ and $r$. It is not hard to see that the ratio $\frac{\mu}{\lambda}$ equals the cross ratio $\frac{|l \cup j| \cdot|j \cup r|}{|l| \cdot|r|}$. Linear transformations preserve this cross-ratio, and the contraction is due to Schwartz inclusion of the image arc in the cone $\mathcal{C}_{n}$.

Remark 2. - The consecutive visits of the $\tilde{J}$ 's in $\rho_{n+1}$ to $\tilde{J}$ 's in $\rho_{n}$ give a direct way to describe $f \mid \omega(0)$ as a substitution shift based on a chain of substitutions $\chi_{n}$. The matrices $A_{n}$ are the associated matrices of the substitutions $\chi_{n}$, cf. $[6,13]$. The proof of unique ergodicity then becomes almost identical to the one given in [6].

Remark 3. - The proof of Proposition 1 can be applied to unicritical complex maps as well. In this case, Yoccoz puzzle pieces will take the role of nice intervals, see Section 4. However, since we have no analogue of the "no wandering interval" result from real dynamics, it is not true in all generality that $\sup \left\{\operatorname{diam}(J) \mid J \in H_{n}\right\} \rightarrow 0$ as $n \rightarrow \infty$. Therefore, Proposition 1 can only 
be used to show that there is a unique invariant probability measure which is measurable with respect to the partition into atoms $\bigcap_{n}\left\{J \in H_{n} \mid x \in J\right\}, x \in \omega(0)$.

Proposition 1 does generalize to the real multimodal case; for the definition of nice intervals and its children in the multimodal setting, we refer to [19].

\subsection{SRB-measures}

For this subsection, we allow $f$ to be a multimodal interval map with a finite set Crit of critical points all of which are non-flat. Assume also that $f$ has only repelling periodic points. Such maps have no wandering intervals (cf. [29]). According to [5,38], the number of ergodic components of Lebesgue measure $m$ is bounded by \# Crit. For each ergodic component, there exists a subset $E$ of full Lebesgue measure, and satisfies exactly one of the following properties:

1. There exists $\varepsilon>0$ such that for any $x \in E$

$$
\limsup _{n \rightarrow \infty} \frac{1}{n} \#\left\{0 \leqslant i<n \mid f^{i}(x) \notin B(\omega(\text { Crit }), \varepsilon)\right\}>0 .
$$

In this case, there is an acip with $E$ as set of typical points, see Proposition 2.

2. For all $\varepsilon>0$ and any $x \in E$

$$
\lim _{n \rightarrow \infty} \frac{1}{n} \#\left\{0 \leqslant i<n \mid f^{i}(x) \in B(\omega(\text { Crit }), \varepsilon)\right\}=1 .
$$

In this case, any possible physical measure is supported in $\omega$ (Crit).

The next proposition shows that under condition (4), an acip $\mu$ exists. As the Lebesgue measure $m(E)>0, \mu$ is automatically physical.

PROPOSITION 2. - Let $f$ be a $C^{3}$ multimodal map with only repelling periodic points, having an ergodic component of Lebesgue measure such that (4) holds for its set $E$ of typical points. Then $f$ has an acip $\mu$, and $\operatorname{supp}(\mu)$ is a finite union of intervals.

Proof. - Take $\varepsilon>0$ such that (4) holds. Since $f$ has no wandering intervals or non-repelling periodic points, $\bigcup_{n} f^{-n}$ (Crit) is dense. For some large $\alpha>0$, take $N$ so large that $P:=$ $\bigcup_{n \leqslant N} f^{-n}$ (Crit) is such that every component of $I \backslash P$ has diameter less than $\frac{\varepsilon}{2 \alpha}$. For at least one component $J$ of $I \backslash P$, with $J \cap B(\omega($ Crit $) ; \varepsilon / 2)=\emptyset$ we have

$$
\limsup _{n \rightarrow \infty} \frac{1}{n} \#\left\{0 \leqslant i<n \mid f^{i}(x) \in J\right\}=: \eta>0
$$

for all $x \in E$. (Note that the same limsup is achieved by all $x \in E$ since $E$ is contained in an ergodic component.) By construction of $P, f^{n}(\partial J) \cap J=\emptyset$ for all $n \geqslant 0$, so $J$ is nice. Therefore, the first return map $F: J \rightarrow J$ has only monotone onto branches $F_{i}: J_{i} \rightarrow J$. Let $\tau_{i}>0$ be such that $F_{i}=f^{\tau_{i}}$. By the choice of $P$, the concentric neighborhood $\alpha J$ of $J$ of length $\alpha|J|$ is disjoint from $\omega$ (Crit). Hence for any interval $J^{\prime}$ and integer $s \geqslant 1$, if $f^{s}: J^{\prime} \rightarrow J$ is monotone onto, then there exists $\hat{J}^{\prime} \supset J^{\prime}$ such that $f^{s}: \hat{J}^{\prime} \rightarrow \alpha J$ is also monotone onto. By Theorem C(2) of [38], there exists $C>0$ independent of $J^{\prime}$ and $s$ such that the distortion of $f^{s} \mid J^{\prime}$ is bounded by $C$ :

$$
\sup _{y_{1}, y_{2} \in J^{\prime}} \frac{D f^{s}\left(y_{1}\right)}{D f^{s}\left(y_{2}\right)} \leqslant C .
$$

Let $H \subset J$ be the set of points on which $F^{k}$ is defined for all $k$. Then $H$ is forward invariant under $F$ and $m(H \cap J) \geqslant m(E \cap J)>0$. Take a density point $x$ of $H$ in $H \cap J$, and let

$4^{\text {e }}$ SÉRIE - TOME $39-2006-\mathrm{N}^{\circ} 3$ 
$F^{k}: J^{k} \rightarrow J$ be the branch at $x$. Then $m\left(J^{k} \cap H\right) / m\left(J^{k}\right) \rightarrow 1$ as $k \rightarrow \infty$, and because $F^{k}$ has distortion bounded uniformly in $k$, we find that $m\left(F^{k}\left(J^{k} \cap H\right)\right) \rightarrow m(J)$ as $k \rightarrow \infty$. Therefore $m(H)=m(J)$. Now the Folklore Theorem (cf. [26]) produces an ergodic $F$-invariant absolutely continuous probability measure $\nu$ with $\operatorname{supp}(\nu)=\bar{J}$ and $\frac{\mathrm{d} \nu}{\mathrm{d} m}$ is bounded and bounded away from 0.

For $x \in H$, define the return time $\tau(x)>0$ such that $F(x)=f^{\tau(x)}(x)$, and let $\tau_{N}(x)=$ $\min \{N, \tau(x)\}$. Then $\tau_{N} \in L^{1}(\nu)$, and by Birkhoff's Ergodic Theorem, $\nu$-a.e. $x \in H$ satisfies

$$
\int \tau_{N} \mathrm{~d} \nu=\lim _{n \rightarrow \infty} \frac{1}{n} \sum_{i=0}^{n-1} \tau_{N}\left(F^{i}(x)\right) \leqslant \liminf _{n \rightarrow \infty} \frac{1}{n} \sum_{i=0}^{n-1} \tau\left(F^{i}(x)\right)=\frac{1}{\eta}<\infty .
$$

This shows that $\eta_{0}:=\int \tau \mathrm{d} \nu<\infty$. Therefore we can pullback $\nu$ to obtain an absolutely continuous $f$-invariant probability measure

$$
\mu(A)=\frac{1}{\eta_{0}} \sum_{k} \sum_{i=0}^{k-1} \nu\left(f^{-i}(A) \cap\{\tau=k\}\right) .
$$

The support of $\mu$ is the forward orbit of $J \subset \bar{E}$. Since $f$ has only repelling periodic orbits and there are no wandering intervals, $\operatorname{supp}(\mu)$ is a finite union of compact intervals. Finally, $\mu$ is physical, because its set $E$ of typical points has positive Lebesgue measure.

We are now in the position to prove Theorem 2.

Proof of Theorem 2. - If Condition (4) holds then Proposition 2 implies that $f$ has an acip $\mu$; its set of typical points $E$ has positive Lebesgue measure, so $\mu$ is physical. If on the other hand (5) holds, then any accumulation point of Cesaro means of Dirac measures $\sum_{i=0}^{n-1} \delta_{f^{i}(x)}$ is an invariant measure supported on $\omega(0)$. But by Proposition $1, f \mid \omega(0)$ is uniquely ergodic. Therefore the invariant measure on $\omega(0)$ is physical.

Remark 4. - For $C^{2}$ non-flat multimodal maps with all periodic points repelling, compact forward invariant sets that are disjoint from Crit are hyperbolic and have zero Lebesgue measure. Therefore each acip contains at least one critical point in its support. It follows from [38, Theorem E] that any critical point interior to the support of an acip cannot be in the support of another physical measure.

For singular physical measures, the situation is different. There are examples where the physical measure is supported on a hyperbolically repelling set, see [16]. It is also possible to construct, for example, a bimodal map on $[0,1]$ with two Cantor attractors, such that the basins of both attractors are dense in $[0,1]$.

\section{No decaying geometry implies low combinatorial complexity}

Throughout this section we consider a $C^{3}$ unimodal map $f:[-1,1] \rightarrow[-1,1]$ with a non-flat critical point located at 0 and with $f(-1)=f(1)=-1$. The constants appearing below are universal in the sense that they only depend on previously introduced constants and the order of the critical point, provided that the intervals involved in the argument are sufficiently small.

Recall that $\alpha I$ denotes the interval of length $\alpha|I|$ that is concentric with $I$.

Let $I$ be a nice interval. Let us denote the first entry domain to $I$ containing $x$ by $\mathcal{L}_{x}(I)$ and inductively define $\mathcal{L}_{x}^{n+1}(I)$ to be $\mathcal{L}_{x}\left(\mathcal{L}_{x}^{n}(I)\right)$. The interval $I$ is called $\delta$-nice, if for each $x \in I \cap \omega(0)$ we have $(1+2 \delta) \mathcal{L}_{x}(I) \subset I$. 
LEMMA 3. - There exists $\delta_{0}>0$ such that if $I \ni 0$ is a nice interval with a non-central return (i.e., with $\left.R_{I}(0) \notin \mathcal{L}_{0}(I)\right)$ then

$$
\left(1+2 \delta_{0}\right) \mathcal{L}_{0}^{2}(I) \subset \mathcal{L}_{0}(I)
$$

Moreover, under the same assumption, for each $\varepsilon>0$ there exists $\eta \in(0,1)$ such that if $\left|\mathcal{L}_{0}(I)\right| \geqslant(1-\eta)|I|$ then

$$
\left|\mathcal{L}_{0}^{2}(I)\right| \leqslant \varepsilon\left|\mathcal{L}_{0}(I)\right|
$$

Proof. - See [27] as well as [38].

An interval $J$ is called a pullback of $I$ if there exist $s>0$ and intervals $J_{0}, \ldots, J_{s}$ with $J_{0}=J$, $J_{s}=I$ and $J_{i}$ a component of $f^{-1}\left(J_{i+1}\right)$ for $i=0, \ldots, s-1$. The order of this pullback is the number of $i \in\{0, \ldots, s-1\}$ so that $J_{i}$ contains a critical point.

LEMma 4. - For any $N$ and $\rho>0$, there exists $\rho^{\prime}>0$ such that if $J$ is a pullback of a nice interval I with order bounded by $N$, and if $(1+2 \rho) J \subset I$, then $J$ is a $\rho^{\prime}$-nice interval. Moreover, $\rho^{\prime} \rightarrow \infty$ as $\rho \rightarrow \infty$.

Proof. - See Lemma 9.7 in [19].

LEMMA 5. - For any $\rho>0, \delta>0$ there exists $r>0$ such that if $I$ is a $\delta$-nice interval and $K_{1} \supsetneq K_{2} \supsetneq \cdots$ are children of $I$, then $I \supset(1+2 \rho) K_{i}$ for $i \geqslant r$.

Proof. - For each $i \geqslant 1$ there exists $s_{i} \in \mathbb{N}$ such that $f^{s_{i}-1}$ maps a one-side neighborhood $T_{i}$ of $f\left(K_{i}\right)$ onto $I$. Clearly, $f^{s_{i}}\left(K_{i+1}\right)$ is contained in a return domain of $I$. By the real Koebe principle, $T_{i}$ contains a definite neighborhood of $f\left(K_{i+1}\right)$ and hence $K_{i}$ contains a definite neighborhood of $K_{i+1}$. The lemma follows.

LEMMA 6. - Assume that $f$ is non-renormalizable and persistently recurrent. For each $\rho>0$ and $\delta>0$, there exists $N=N(\rho, \delta)$ with the following property. Let $I$ be a $\delta$-nice interval and let $\Gamma$ be its smallest child. Let $I:=I^{0} \supset I^{1} \supset I^{2} \supset \cdots$ the principal nest corresponding to $I$, i.e., $I^{i}=\mathcal{L}_{0}\left(I^{i-1}\right)$ for $i \geqslant 1$ and let $m$ be a positive integer such that $R_{I^{i}}(0)=R_{I^{0}}(0)$ for $i=0, \ldots, m-1$. If there exists $N^{\prime} \geqslant N$ and $z \in \omega(0)$ such that $R_{I}^{j}(z) \in\left(I \backslash I^{m}\right)$ for $j=0, \ldots, N^{\prime}$ and at least $N$ of these points are in $I \backslash I^{1}$, then $(1+2 \rho) \Gamma \subset I$.

Proof. - Let us show that $I$ has at least $N$ children. Write $R:=R_{I}$ and let $n_{1}<n_{2}<$ $\cdots<n_{N} \leqslant N^{\prime}$ be so that $R^{n_{i}}(z) \in I \backslash I^{1}$. Since $z, \ldots, R^{N^{\prime}}(z) \notin\left(I \backslash I^{m}\right), \quad R^{n_{i+1}}$ maps a neighborhood $J_{i}$ of $z$ diffeomorphically onto $I$. (Here we use that $R$ maps a component of $I^{i} \backslash I^{i+1}, 1 \leqslant i \leqslant m-1$, diffeomorphically onto a component of $I^{i-1} \backslash I^{i}$.) It follows that $K_{i}:=\mathcal{L}_{0}\left(J_{i}\right)$ is a child of $I$. Since $J_{1} \supsetneq J_{2} \supsetneq \cdots \supsetneq J_{N}$ we also have $K_{1} \supsetneq K_{2} \supsetneq \cdots \supsetneq K_{N}$, i.e., $I$ has at least $N$ children. So if we let $r$ be the integer associated to $\delta$ and $\rho$ from Lemma 5 then the conclusion of the lemma holds if $N \geqslant r$.

PROPOSITION 3.- Assume that $f$ is non-renormalizable and persistently recurrent. Let $\Gamma^{0} \supset \Gamma^{1} \supset \cdots \ni 0$ be a sequence of nice intervals such that $\Gamma^{n+1}$ is the smallest child of $\Gamma^{n}$, $n=0,1, \ldots$ For each $\rho>0$ there exists $C>0$ so that for any $n \geqslant 2$, if the combinatorial complexity $\mathcal{G}_{n} \geqslant C$, then $\Gamma^{n+2}$ is $\rho$-nice.

Proof. - By Lemma 4, there exists $\tau=\tau(\rho)>0$ such that $\Gamma^{n+2}$ is $\rho$-nice if $\left|\Gamma^{n+2}\right| /\left|\Gamma^{n}\right|<\tau$ since $\Gamma^{n+2}$ is a pull back of $\Gamma^{n}$ of order 2 . Assuming that $\left|\Gamma^{n+2}\right| /\left|\Gamma^{n}\right| \geqslant \tau$, let us show that $\mathcal{G}_{n}$ cannot be too large.

$4^{\text {e }}$ SÉRIE - TOME $39-2006-\mathrm{N}^{\circ} 3$ 
Let $I^{0}=\Gamma^{n}$ and let $I^{i}$ be the corresponding principal nest. Let $i(0)=0$ and $i(1)<i(2)<\cdots$ be all the positive integers such that $R_{i(j)-1}(0) \notin I^{i(j)}$. Choose $r$ so that $I^{r+1} \subset \Gamma^{n+1} \subsetneq I^{r}$, and let $q$ be maximal with $i(q) \leqslant r$. By Lemma 3, $q$ is bounded from above by a constant $q(\tau)$.

CLAIM. - There exists $\delta=\delta(\tau)>0$ such that $I^{i(j)}$ is a $\delta$-nice interval for all $0 \leqslant j \leqslant q$.

First let us consider the case $1 \leqslant j \leqslant q$. As $I^{i(j)+1} \supset \Gamma^{n+2}$ we have $\left|I^{i(j)+1}\right| /\left|I^{i(j)}\right| \geqslant \tau$. Therefore, by the second statement of Lemma 3, there exists $\eta$ (depending on $\varepsilon:=\tau$ ) so that $\left|I^{i(j)}\right| /\left|I^{i(j)-1}\right| \leqslant(1-\eta)$. By Lemma 4, there exists $\delta=\delta(\tau)>0$ such that $I^{i(j)}$ is $\delta$-nice. Now let us consider the case $j=0$. Again by Lemma 4, it suffices to show that $I^{0}=\Gamma^{n}$ is well inside $\Gamma^{n-2}$. So define $\hat{I}^{0}=\Gamma^{n-2}$ and for $i \geqslant 1, \hat{I}^{i}=\mathcal{L}_{0}\left(\hat{I}^{i-1}\right)$, and let $m \geqslant 1$ be minimal such that $R_{\hat{I}^{m-1}}(0) \notin \hat{I}^{m}$. Then, for $i=1, \ldots, m-1, R_{\hat{I}^{0}} \mid\left(\hat{I}^{i} \backslash \hat{I}^{i+1}\right)$ is a diffeomorphism on each component of $\hat{I}^{i} \backslash \hat{I}^{i+1}$ and its range contains $\left(\hat{I}^{i-1} \backslash \hat{I}^{i}\right)$. Since $\hat{I}^{0} \cap \omega(0)$ contains a point outside $\hat{I}^{1}$, it follows that there exists a child of $\hat{I}^{0}$ which is contained in $\hat{I}^{m}$. In particular, $\hat{I}^{m} \supset \Gamma^{n-1}$, and hence $\hat{I}^{m+1} \supset \Gamma^{n}$. By the first statement of Lemma 3, it follows that

$$
\left(1+2 \delta_{0}\right) \Gamma^{n} \subset\left(1+2 \delta_{0}\right) \hat{I}^{m+1} \subset \hat{I}^{m} \subset \Gamma^{n-2} .
$$

This completes the proof of the claim.

Now let $N=N\left(\tau^{-1}, \delta\right)$ be as in Lemma 6. Let us show that $\mathcal{G}_{n} \leqslant N^{q+1}$. To this end, let $y \in \omega(0) \cap \Gamma^{n}$ be such that $\mathcal{G}_{n}(y)=\mathcal{G}_{n}$ and let $s \geqslant 0$ be minimal such that $f^{s}(y) \in \Gamma^{n+1}$. Note that if $0<i<r$ and $I^{i}$ is central, i.e., $R_{I^{i-1}}(0) \in I^{i}$, then $R_{I^{i-1}}$ maps $I^{i} \backslash I^{i+1}$ into $I^{i-1} \backslash I^{i}$, so in the definition of combinatorial complexity, visits to $I^{i} \backslash I^{i+1}$ do not contribute to $\mathcal{G}_{n}$. Therefore

$$
\#\left\{0 \leqslant k<s \mid f^{k}(y) \in \bigcup_{j=0}^{q} I^{i(j)} \backslash I^{i(j)+1}\right\} \geqslant \mathcal{G}_{n} .
$$

For $j \geqslant 0$, let

$$
\nu(j)=\#\left\{0 \leqslant k<s \mid f^{k}(x) \in I^{i(j)} \backslash I^{i(j)+1}\right\} .
$$

Note that $\nu(q+1)=0$.

Let us show that for any $0 \leqslant j \leqslant q, \nu(j) \leqslant(\nu(j+1)+1)(N-1)$. Indeed, otherwise, there exists $0 \leqslant s^{\prime}<s$ such that the orbit $\left\{f^{k}(y)\right\}_{k=s^{\prime}}^{s}$ visits $I^{i(j)} \backslash I^{i(j)+1}$ at least $N$ times before it enters $I^{i(j+1)}$. By Lemma 6, this, together with the claim above, implies that if $K_{j}$ is the last child of $I^{i(j)}$ then $\left(1+2 \tau^{-1}\right) K_{j} \subset I^{i(j)}$. Noticing $I^{i(j)} \supset \Gamma^{n+1}$, we have $K_{j} \supset \Gamma^{n+2}$. Therefore $\left(1+2 \tau^{-1}\right) \Gamma^{n+2} \subset I^{i(j)} \subset \Gamma^{n}$, contradicting the hypothesis $\left|\Gamma^{n+2}\right| /\left|\Gamma^{n}\right| \geqslant \tau$.

It follows that $\nu(j) \leqslant N^{q-j+1}-N^{q-j}$ for all $0 \leqslant j \leqslant q$. So $\mathcal{G}_{n} \leqslant \sum_{i=0}^{q} \nu(j) \leqslant N^{q+1}$.

Proof of Theorem 3. - If $f \in \mathcal{F}_{r}^{0}$ does not have low combinatorial complexity, then $\mathcal{G}_{n} \rightarrow \infty$. By Proposition 3 it follows that $f \in \mathcal{D G}$ in this case. Therefore any map in $\mathcal{F}_{r}^{0} \backslash \mathcal{D G}$ must have low combinatorial complexity.

\subsection{The proof of Theorem 5}

We will prove Theorem 5 by a random walk argument, using $\alpha_{k}$ to indicate the state after the $k$-th step in the walk. The Lebesgue measure (normalized on $\left[f(0), f^{2}(0)\right]$ ) will be denoted as $m$.

Proof of Theorem 5. - If $f$ is infinitely renormalizable, then $f \mid \omega(0)$ is uniquely ergodic [15] and there is nothing to prove. If $f$ is finitely renormalizable, then by passing to the "deepest renormalization", we can assume that $f$ is not renormalizable anymore. It remains to consider 
the case that $f$ has a wild attractor. By [21] (see also [7]), $f$ is persistently recurrent. Let us assume that the combinatorial complexity of $f$ is not low, so in particular, $\lim _{n \rightarrow \infty} \mathcal{G}_{n}=\infty$. Proposition 3 states that, given $\rho>0$, there exists $n_{0}$ such that $\Gamma^{n}$ is $\rho$-nice, and hence $(1+2 \rho) \mathcal{L}_{0}\left(\Gamma^{n}\right) \subset \Gamma^{n}$ for all $n \geqslant n_{0}$. We will use a by now standard random walk argument on an induced map, see e.g. [9], to prove that a wild attractor cannot exist after all, contradicting the assumption that the combinatorial complexity of $f$ is not low. Therefore Theorem 2 implies that $f \mid \omega(0)$ is uniquely ergodic.

We start by defining an inducing scheme. Let $R_{n}:\left[f(0), f^{2}(0)\right] \rightarrow \Gamma^{n}$ be the first return/entry map to $\Gamma^{n}$; it is defined $m$-a.e., and, except for the central branch $\mathcal{L}_{0}\left(\Gamma^{n}\right)$, all branches are onto $\Gamma^{n}$. Recall that for each $n \geqslant 1$, there exists $s_{n+1}$ such that $f^{s_{n+1}-1}$ maps a onesided neighborhood $\widetilde{\Gamma}^{n+1}$ of $f\left(\Gamma^{n+1}\right)$ monotonically onto $\Gamma^{n}$. Let $j=j_{n} \geqslant 0$ be minimal such that $R_{n}^{j} \circ f^{s_{n+1}}(0) \in \mathcal{L}_{0}\left(\Gamma^{n}\right)$, and let $t \geqslant 0$ be such that $R_{n}^{j} \circ f^{s_{n+1}}(0)=f^{s_{n+1}+t}(0)$. Let $V_{n+1}$ be the maximal neighborhood of 0 such that $f^{s_{n+1}+t}\left(V_{n+1}\right) \subset \mathcal{L}_{0}\left(\Gamma^{n}\right)$. Then $\mathcal{L}_{0}\left(\Gamma^{n+1}\right) \supset V_{n+1} \supset \Gamma^{n+2}$. The first inclusion is immediate, and the second follows because otherwise $f^{s_{n+1}+t}\left(\Gamma^{n+2}\right)$ contains a boundary point of $\mathcal{L}_{0}\left(\Gamma^{n}\right)$, and hence cannot be mapped monotonically onto $\Gamma^{n+1}$.

Let $U_{n+1} \supset V_{n+1}$ be the maximal neighborhood such that $f^{s_{n+1}+t}\left(U_{n+1}\right) \subset \Gamma^{n}$. Note that $U_{n+1} \subset \mathcal{L}_{0}\left(\Gamma^{n+1}\right)$. For $n \geqslant n_{0},(1+2 \rho) \mathcal{L}_{0}\left(\Gamma^{n}\right) \subset \Gamma^{n}$. As $f^{s_{n+1}+t} \mid U_{n+1}$ is the composition of $f$ with a map of uniformly bounded distortion, there exists $\rho^{\prime}=\mathcal{O}\left(\rho^{1 / \ell}\right)$ such that $\left(1+2 \rho^{\prime}\right)\left(V_{n+1}\right) \subset U_{n+1}$ and $\rho^{\prime} \rightarrow \infty$ as $\rho \rightarrow \infty$.

We define the induced map $Q$ on $\Gamma^{n+1} \backslash \Gamma^{n+2}$ as follows:

- If $x \in V_{n+1} \backslash \Gamma^{n+2}$, then let $t^{\prime} \geqslant t$ be minimal such that $f^{s_{n+1}+t^{\prime}}(x) \in \Gamma^{n+2}$, and let $Q(x)=f^{s_{n+1}+t^{\prime}}(x)$. Note that there exists a neighborhood $U_{x} \ni x$ such that $f^{s_{n+1}+t^{\prime}}$ maps $U_{x}$ monotonically onto $\Gamma^{n+2}$.

- If $x \in \Gamma^{n+1} \backslash V_{n+1}$, then let $k=k(x) \in\left\{0,1, \ldots, j_{n}-1\right\}$ be the minimal integer such that $R_{n}^{k} \circ f^{s_{n+1}}(x)$ and $R_{n}^{k} \circ f^{s_{n+1}}(0)$ lie in different return domains of $R_{n}$. If the return domain containing $R_{n}^{k} \circ f^{s_{n+1}}(x)$ is $\mathcal{L}_{0}\left(\Gamma^{n}\right)$, then $Q(x)=R_{n}^{k} \circ f^{s_{n+1}}(x)$. In this case there exists a neighborhood $U_{x} \ni x$ such that $R_{n}^{k} \circ f^{s_{n+1}}$ maps $U_{x}$ monotonically onto $\mathcal{L}_{0}\left(\Gamma^{n}\right)$. Otherwise, $Q(x)=R_{n}^{k+1} \circ f^{s_{n+1}}(x)$, and there exists a neighborhood $U_{x} \ni x$ such that $R_{n}^{k+1} \circ f^{s_{n+1}}$ maps $U_{x}$ monotonically onto $\Gamma^{n}$.

In this manner $Q$ is defined for $m$-a.e. $x \in \Gamma^{n+1} \backslash \Gamma^{n+2}$ and the monotone image of the corresponding neighborhood $U_{x}$ is one of $\Gamma^{n}, \mathcal{L}_{0}\left(\Gamma^{n}\right)$ or $\Gamma^{n+2}$. Moreover, any two neighborhoods $U_{x}$ or $U_{y}$ are either disjoint or coincide.

Repeating this construction for all $n$, we obtain a Markov induced map $Q$ which preserves the partition given by the boundary points of the intervals $\Gamma^{n}$ and $\mathcal{L}_{0}\left(\Gamma^{n}\right)$.

To describe the random walk, let $\alpha_{k}=n$ if $Q^{k}(x) \in \Gamma^{n} \backslash \Gamma^{n+1}$. The $\alpha_{k}$ can be considered as random variable with the following conditional probabilities. For $n>n_{0}$ we have

$$
\begin{aligned}
P\left(\alpha_{k+1}=n-1 \mid \alpha_{k}=n\right) & :=\frac{m\left(\alpha_{k}=n \text { and } \alpha_{k+1}=n-1\right)}{m\left(\alpha_{k}=n\right)} \\
& \geqslant 1-\mathcal{O}\left(1 / \rho^{\prime}\right) .
\end{aligned}
$$

Indeed, if $\alpha_{k}(x)=n$, then there is a neighborhood $W_{x}$ of $x$ such that $Q^{k}$ maps $W_{x}$ monotonically onto $\Gamma^{n}$ or $\mathcal{L}_{0}\left(\Gamma^{n}\right)$. Given $y \in W_{x}$, there are three cases to consider:

- $Q^{k}(y) \in V_{n}$ (in this case $\left.Q^{k+1}(y) \in \Gamma^{n+1}\right)$. As $\left(1+2 \rho^{\prime}\right) V_{n} \subset U_{n} \subset \mathcal{L}_{0}\left(\Gamma^{n}\right)$, $m\left(\left\{y \in W_{x} \mid Q^{k}(y) \in V_{n}\right\}\right)=\mathcal{O}\left(1 / \rho^{\prime}\right) m\left(W_{x}\right)$.

- $Q^{k}(y) \in U$ where $U$ is a domain of $Q$ such that $Q(U)=\Gamma^{n-1}$. Because $\left|\Gamma^{n}\right| /\left|\Gamma^{n-1}\right|=$ $\mathcal{O}(1 / \rho)$,

$$
m\left(\left\{y \in W_{x} \mid Q^{k}(y) \in U, Q^{k+1}(y) \in \Gamma^{n}\right\}\right)=\mathcal{O}(1 / \rho) m\left(W_{x} \cap Q^{-k}(U)\right) .
$$

$4^{\text {e }}$ SÉRIE - TOME $39-2006-\mathrm{N}^{\circ} 3$ 
- $Q^{k}(y) \in U^{\prime}$ where $U^{\prime}$ is a domain of $Q$ such that $Q\left(U^{\prime}\right)=\mathcal{L}_{0}\left(\Gamma^{n-1}\right)$. Let $p$ be such that $Q\left|U^{\prime}=f^{p}\right| U^{\prime}$. In this case there is a neighborhood $U^{\prime \prime}$ adjacent to $U^{\prime}$ such that $f^{p}$ maps $U^{\prime \prime}$ monotonically onto a component of $\Gamma^{n-1} \backslash \mathcal{L}_{0}\left(\Gamma^{n-1}\right)$. As $(1+2 \rho) \mathcal{L}_{0}\left(\Gamma^{n-1}\right) \subset \Gamma^{n-1}$,

$$
m\left(\left\{y \in W_{x} \mid Q^{k}(y) \in U^{\prime}\right\}\right)=\mathcal{O}(1 / \rho) m\left(\left\{y \in W_{x} \mid Q^{k}(y) \in U^{\prime \prime}\right\}\right) .
$$

Combining these estimates and adding over all domains $W_{x}$ with $Q^{k}\left(W_{x}\right) \subset \Gamma^{n}$, we arrive at (6). A similar argument gives for $r \geqslant 1$,

$$
\begin{aligned}
P\left(\alpha_{k+1}=n+r \mid \alpha_{k}=n\right) & =\frac{m\left(\alpha_{k}=n \text { and } \alpha_{k+1}=n+r\right)}{m\left(\alpha_{k}=n\right)} \\
& \leqslant \mathcal{O}\left(\left|\Gamma^{n+r}\right| /\left|\Gamma^{n}\right|\right)=\mathcal{O}\left(\rho^{-r}\right) .
\end{aligned}
$$

Therefore, the drift of the random walk is

$$
\mathbb{E}\left(\alpha_{k+1}-n \mid \alpha_{k}=n\right)=\sum_{r \geqslant-1} r P\left(\alpha_{k+1}=n+r \mid \alpha_{k}=n\right) \leqslant-\frac{1}{2},
$$

for $\rho$ and hence $\rho^{\prime}$ sufficiently large and $n>n_{0}(\rho)$. A similar computation shows that the variance is bounded as well:

$$
\begin{aligned}
d \operatorname{Var}\left(\alpha_{k+1}-n \mid \alpha_{k}=n\right) & \leqslant \mathbb{E}\left(\left(\alpha_{k+1}-n\right)^{2} \mid \alpha_{k}=n\right) \\
& =\sum_{r \geqslant-1} r^{2} P\left(\alpha_{k+1}=n+r \mid \alpha_{k}=n\right) \\
& <1+\sum_{r \geqslant 1} r^{2} \mathcal{O}\left(\rho^{-r}\right)<\infty .
\end{aligned}
$$

Hence we can apply the random walk argument from [9] to conclude that $\liminf _{k \rightarrow \infty} \alpha_{k}<\infty$ for $m$-a.e. $x$, and this excludes the existence of a wild attractor.

\section{Yoccoz puzzle}

Let us consider the family $f_{c}(z)=z^{\ell}+c$ parametrized by $c \in \mathbb{C}$. By definition, the filled Julia set $K_{c}$ of $f_{c}$ is the complement of the open set

$$
A_{c}(\infty)=\left\{z \in \mathbb{C} \mid f_{c}^{n}(z) \rightarrow \infty \text { as } n \rightarrow \infty\right\},
$$

which is the attracting basin of infinity. The Green function

$$
G_{c}: \mathbb{C} \rightarrow \mathbb{R}_{+}=\{t \geqslant 0\}, \quad z \mapsto \lim _{n \rightarrow \infty} \frac{1}{\ell^{n}} \log ^{+}\left|f_{c}^{n}(z)\right|,
$$

is a subharmonic function vanishing exactly on the filled Julia set $K_{c}$. The classical Böttcher Theorem provides us a unique conformal representation

$$
B_{c}:\left\{z \mid G_{c}(z)>G_{c}(0)\right\} \rightarrow\left\{z|| z \mid>r_{c}\right\}, \quad \text { where } \log r_{c}=G_{c}(0),
$$

such that $B_{c}$ is tangent to the identity at infinity $B_{c} \circ f_{c}=\left(B_{c}\right)^{\ell}$. 
The Green function is equal to $\log \left|B_{c}\right|$ on the domain of $B_{c}$. The level curve $\left\{G_{c}(z)=r\right\}$, $r>0$ is called the equipotential curve of level $r$, and denoted by $E_{c}(r)$. The external ray of angle $t \in \mathbb{R} / \mathbb{Z}$ is the gradient curve of $G_{c}$ stemming from infinity with the angle $t$ (measured via the Böttcher coordinate $B_{c}$ ), and denoted by $R_{c}(t)$. When $c$ is contained in the Multibrot set

$$
\mathcal{M}=\left\{c \in \mathbb{C} \mid K_{c} \text { is connected }\right\}
$$

the map $B_{c}$ is defined in the whole complement $A_{c}(\infty)$ of the filled Julia set $K_{c}$, and so $R_{c}(t)=B_{c}^{-1}\left(\left\{r e^{2 \pi i t} \mid r>1\right\}\right)$. In this case, any external ray $R_{c}(t)$ with $t$ rational has a well defined landing point $\lim _{r \rightarrow 1^{+}} B_{c}^{-1}\left(r e^{2 \pi i t}\right)$ which is contained in the Julia set $\partial K_{c}$; vice versa, a repelling or parabolic point is the common landing point of finitely many external rays with rational angle. When $K_{c}$ is disconnected, provided that $\arg B_{c}(c) \neq \ell^{k} t$ for all $k \geqslant 1$, the external ray $R_{c}(t)$ is still a smooth curve joining infinity and $\partial K_{c}$, so each point in $R_{c}(t)$ has a uniquely defined potential.

For every $c \in \mathbb{C}$, the domain of $B_{c}$ contains the critical value $c$ of $f_{c}$ so that $B_{c}(c)$ is well defined. By [11], the set $\mathcal{M}$ is connected and the map $\Phi(c)=B_{c}(c)$ defines a conformal map from $\mathbb{C} \backslash \mathcal{M}$ onto $\mathbb{C} \backslash \overline{\mathbb{D}}$. As in the dynamical plane, the parameter (external) ray of angle $t \in \mathbb{R} / \mathbb{Z}$ is the set

$$
\mathcal{R}(t)=\Phi^{-1}\left(\left\{r e^{2 \pi i t} \mid r>1\right\}\right),
$$

and the equipotential of level $r>0$ is the closed curve

$$
\mathcal{E}(r)=\{c \in \mathbb{C} \backslash \mathcal{M}|\log | \Phi(c) \mid=r\} .
$$

Let $\mathcal{H}$ denote the component of the interior of $\mathcal{M}$ which contains 0 . This is the region where $f_{c}$ has an attracting fixed point. For $c_{0} \in(\mathcal{M} \backslash \mathcal{H}) \cap \mathbb{R}, f_{c_{0}}$ has an orientation reversing fixed point $\alpha_{c_{0}}$ in $\mathbb{R}$. There exist exactly two external rays $R_{c_{0}}\left(t^{-}\right), R_{c_{0}}\left(t^{+}\right)$landing at $\alpha_{c_{0}}$, see Lemma 5.2 in [19]. These two external rays are symmetric to each other with respect to the real axis, and permuted by $f_{c}$ :

$$
\ell t^{-}=t^{+}, \quad \ell t^{-}=t^{+}, \quad \bmod 1 .
$$

Arguing as in Theorem 2.1 in [30], the corresponding dynamical rays $\mathcal{R}\left(t^{-}\right)$and $\mathcal{R}\left(t^{+}\right)$land at a common point $\gamma \in \mathbb{R}$. The configuration $\mathcal{R}\left(t^{-}\right) \cup \mathcal{R}\left(t^{+}\right) \cup\{\gamma\}$ cuts the parameter plane into two connected components, and we use $\mathcal{W}$ to denote the one which does not contain 0 (the $1 / 2$-wake). The set $\mathcal{W}$ consists of all $c$ for which $f_{c}$ has a repelling fixed point $\alpha_{c}$ at which the external rays $R_{c}\left(t^{+}\right)$and $R_{c}\left(t^{-}\right)$land. In particular,

$$
\mathcal{W} \supset(\mathcal{M} \backslash \mathcal{H}) \cap \mathbb{R} \ni c_{0} .
$$

\subsection{Yoccoz puzzle}

Now let us recall the definition of Yoccoz puzzle for $c \in \mathcal{W}$. Let $X_{c}^{n}=\{z \in \mathbb{C} \mid$ $\left.G_{c}(z)<1 / \ell^{n}\right\}$. By definition, the Yoccoz puzzle of $f_{c}$ is the following sequence of graphs:

$$
\begin{gathered}
S_{c}^{0}=\partial X_{c}^{0} \cup\left(X_{c}^{0} \cap \bigcup_{t \in\left\{t^{+}, t^{-}\right\}} \overline{R_{c}(t)}\right), \\
S_{c}^{n}=f_{c}^{-n} S_{c}^{0}, \quad n=1,2, \ldots
\end{gathered}
$$

A component of $X_{c}^{n} \backslash S_{c}^{n}=f_{c}^{-n}\left(X_{c}^{0} \backslash S_{c}^{0}\right)$ will be called a puzzle piece of depth $n$. A puzzle piece of depth $n$ which contains a point $z$ will be denoted by $P_{c}^{n}(z)$. For $n \geqslant 1$, the $f$-image of

$4^{\text {e }}$ SÉRIE - TOME $39-2006-\mathrm{N}^{\circ} 3$ 
a puzzle piece of depth $n$ is a puzzle piece of depth $n-1$ and each puzzle piece of depth $n$ is contained in a puzzle piece of depth $n-1$.

Definition 2. - Let $m>n \geqslant 0$ be integers. We say that $P_{c}^{m}(0)$ is a child of $P_{c}^{n}(0)$ if $f^{m-n-1}: P_{c}^{m-1}(c) \rightarrow P_{c}^{n}(0)$ is a conformal map.

LemmA 7. - Assume that $c \in \mathcal{W} \cap \mathbb{R}$ is such that $f_{c}$ is non-renormalizable. Then $P_{c}^{2}(c) \Subset$ $P_{c}^{1}(c)$.

Proof. - Otherwise, $P_{2}(c)$ contains $-\alpha_{c}$ in its closure. As $c \in \mathbb{R}$, this implies that $P_{2}(0) \cap \mathbb{R}$ is a periodic interval of period 2 , contradicting that $f_{c}$ is non-renormalizable.

\subsection{First return maps}

Consider a map $f=f_{c}$ with $c \in \mathcal{W}$. Let $V$ be a puzzle piece which contains 0 . Let $D(V)=$ $\left\{z \in \mathbb{C}: \exists k \geqslant 1\right.$ such that $\left.f^{k}(z) \in V\right\}$. The first return map $g_{V}$ is defined as follows: for each $z \in D(V) \cap V$, if $k \geqslant 1$ is the return time of $z$ to $V$, i.e., the minimal $k \geqslant 1$ such that $f^{k}(z) \in V$, then $g_{V}(z)=f^{k}(z)$. It is well-known that the return time is constant on each component $P$ of $D(V) \cap V$ and that $g_{V} \mid P$ is conformal if $P \not \supset 0$ and $\ell$-to- 1 otherwise. If $0 \in D(V)$, and $V$ is strictly nice: $f^{k}(\partial V) \cap \bar{V}=\emptyset$ for all $k \geqslant 1$, then the first return map $g_{V}$ is an R-map as defined below.

Definition 3. - Let $V, U_{j}, j=0,1, \ldots$, be Jordan disks in $\mathbb{C}$ such that the sets $\overline{U_{j}}$ are pairwise disjoint and contained in $V$. A holomorphic map $g: \bigcup_{j=0}^{\infty} U_{j} \rightarrow V$ is called an $R$-map (where "R" stands for "return") if the following hold:

- $g: U_{0} \rightarrow V$ is an $\ell$-to-1 proper map with a unique critical point at 0 ,

- for all $i \geqslant 1, g: U_{j} \rightarrow V$ is conformal and surjective.

$U_{0}$ is usually called the central and also the critical domain of $R$. The renormalization $\mathcal{L} g$ of $R$ is the first return map of $g$ to $U_{0}$. Note that $\mathcal{L} g$ is again an R-map provided that $g^{k}(0) \in U_{0}$ for some $k \geqslant 1$.

The following is a lemma which we shall need later.

For an R-map $g: \bigcup_{i} U_{i} \rightarrow V$ define

$$
\bmod (g)=\bmod \left(V \backslash \bar{U}_{0}\right), \quad \bmod ^{\prime}(g)=\inf \left\{\bmod \left(V \backslash \overline{U_{i}}\right) \mid i \geqslant 1\right\} .
$$

LEMma 8. - Let $g: \bigcup U_{i} \rightarrow V$ be an $R$-map. Let $W$ be a return domain to $U_{0}$ (under $g$ ) and let $s$ be the positive integer such that $\mathcal{L} g\left|W=g^{s}\right| W$. Then

$$
\bmod \left(U_{0} \backslash \bar{W}\right) \geqslant \frac{1}{\ell}\left((s-1) \bmod ^{\prime}(g)+\bmod (g)\right) .
$$

Proof. - For each $1 \leqslant j \leqslant s$, let $i_{j}$ be such that $U_{i_{j}} \supset g^{j}(W)$. Then $i_{j} \neq 0$ for all $1 \leqslant j \leqslant s-1$ and $i_{s}=0$. Let $Q_{j}$ be the component of $g^{-j}(V)$ containing $g(W)$ for $j=0,1, \ldots, s$. Then $W=g^{-1}\left(Q_{s}\right)$. For any $j \leqslant s-1, g^{j}:\left(Q_{j}, Q_{j+1}\right) \rightarrow\left(V, U_{i_{j+1}}\right)$ is a conformal map. So

$$
\begin{aligned}
\bmod \left(V \backslash Q_{s}\right) & \geqslant \sum_{j=0}^{s-1} \bmod \left(Q_{j} \backslash Q_{j+1}\right) \\
& =\sum_{j=0}^{s-1} \bmod \left(V \backslash U_{i_{j+1}}\right) \geqslant(s-1) \ell \bmod ^{\prime}(g)+\bmod (g) .
\end{aligned}
$$

Since $\bmod \left(U_{0} \backslash W\right) \geqslant \bmod \left(V \backslash Q_{s}\right) / \ell$, the lemma follows. 


\subsection{Holomorphic motion}

Definition 4. - A holomorphic motion of a set $X \subset \mathbb{C}$ over a complex manifold $D$ is a map

$$
\mathbf{h}: D \times X \rightarrow D \times \mathbb{C}, \quad(\lambda, z) \mapsto\left(\lambda, h_{\lambda}(z)\right),
$$

which satisfies the following properties:

- for any $\lambda \in D, h_{\lambda}: X \rightarrow \mathbb{C}$ is injective;

- for any $z \in X, \lambda \mapsto h_{\lambda}(z)$ is holomorphic;

- $h_{*}=\operatorname{id}_{X}$ for some $* \in D$.

We shall also say that $\mathbf{h}$ is a holomorphic motion of $X$ over $(D, *)$.

Throughout the remainder of the paper we shall call a proper open subset of $\mathbb{C}$ which is homeomorphic to a disk, a topological disk.

Optimal $\lambda$-LEMma (Slodkowski [37]). - Let $D \subset \mathbb{C}$ be a topological disk and let $c_{0} \in D$. Given any holomorphic motion $\mathbf{h}$ of a set $X \subset \mathbb{C}$ over $\left(D, c_{0}\right)$, there exists a holomorphic motion $\tilde{\mathbf{h}}$ of $\mathbb{C}$ over $\left(D, c_{0}\right)$ such that $\tilde{\mathbf{h}} \mid D \times X=\mathbf{h}$. Moreover, $\tilde{h}_{c}$ is a $K(r)$-qc map, where $r$ is the hyperbolic distance between $c$ and $c_{0}$ in $D$ and $\lim _{r \rightarrow 0} K(r)=1$.

Recently, E. Chirka provided a new proof of this deep result. We shall use the terminology tube for a holomorphic motion $\mathbf{h}$ of a Jordan curve $\gamma$ over a Jordan disk $D \subset \mathbb{C}$. A holomorphic motion $\mathbf{h}$ of a closed Jordan disk $\bar{V} \subset \mathbb{C}$ over another Jordan disk $D \subset \mathbb{C}$ will be called a filled tube. A filled tube is called proper if $\mathbf{h}$ extends to a homeomorphism from $(D \times \bar{V}) \cup(\partial D \times \partial V)$ into $\mathbb{C}^{2}$.

Given a filled tube $\mathbf{h}: D \times \bar{V} \rightarrow D \times \mathbb{C}$, a holomorphic map $\varphi: D \rightarrow \mathbb{C}$ will be called a diagonal of $\mathbf{h}$ if the following hold:

- $\varphi(c) \in h_{c}(V)$ for all $c \in D$,

- $\varphi$ has a continuous extension to $\bar{D}$, and

- $c \mapsto h_{c}^{-1} \circ \varphi(c)$ defines a homeomorphism from $\partial D$ onto $\partial V$.

By the Argument Principle, for each $z \in V$, the equation $h_{c}(z)=\varphi(c)$ has a unique solution in $D$. See [22].

LEMMA 9. - There exists $M>0$ with the following property. Let $D \subset \mathbb{C}$ be a Jordan disk. Let $V \ni U$ be Jordan disks in $\mathbb{C}$ with $\bmod (V \backslash \bar{U})>3 M$. Let $\mathbf{h}: D \times \bar{V} \rightarrow D \times \mathbb{C}$ be a proper filled tube and let $\varphi: D \rightarrow \mathbb{C}$ be a diagonal of $\mathbf{h}$. Assume that for each $c \in D$, there exists a 3-qc map $\hat{h}_{c}: \mathbb{C} \backslash U \rightarrow h_{c}(\mathbb{C} \backslash U)$ which coincides with $h_{c}$ on $\partial V \cup \partial U$. Then $D^{\prime}=\left\{c \in D \mid h_{c}^{-1}(\varphi(c)) \in U\right\}$ is a topological disk, and

$$
\bmod \left(D \backslash \overline{D^{\prime}}\right) \geqslant \frac{1}{3} \bmod (V \backslash \bar{U})-M .
$$

Proof. - The proof follows the idea in Section 4.3 of [22]. We shall use the following wellknown fact: there exists a positive constant $M_{0}>0$ such that if $U \Subset V \subset \mathbb{C}$ are Jordan disks with $\bmod (V \backslash \bar{U})>M_{0}$, then for any $z_{0} \in U$, there exists a round annulus $A\left(z_{0} ; r, R\right):=$ $\left\{r<\left|z-z_{0}\right|<R\right\} \subset V \backslash \bar{U}$ such that $\bmod \left(A\left(z_{0} ; r, R\right)\right)(=\log R / r) \geqslant \bmod (V \backslash \bar{U})-M_{0}$, see [25].

Without loss of generality, we may assume that $0 \in U, 1 \in \partial V$, and that $\mathbb{D} \subset V$. By considering the pre-composition of $h_{c}$ with an affine motion $g_{c}(z)=a_{c} z+b_{c}$, we may assume $h_{c}(z)=z$ for each $c \in D$ and $z \in\{0,1\}$.

Assuming that $m:=\bmod (V \backslash \bar{U})>3 M_{0}$ is large enough, let us prove that there exist $0<r<R<1$ such that

$4^{\text {e }}$ SÉRIE - TOME $39-2006-\mathrm{N}^{\circ} 3$ 
- $D \times A(0 ; r, R) \subset \mathbf{h}(D \times(V \backslash \bar{U}))$;

- $\log (R / r) \geqslant m / 3-M_{1}$, where $M_{1}$ is a constant.

Indeed, by compactness of 3-qc maps there exists a constant $R \in(0,1)$ such that $\left|h_{c}(z)\right|=$ $\left|\hat{h}_{c}(z)\right|>R$ for any $z \in \partial V$ and $c \in D$. Since

$$
\bmod \left(h_{c}(V \backslash \bar{U})\right)=\bmod \left(\hat{h}_{c}(V \backslash \bar{U})\right) \geqslant m / 3,
$$

and since $h_{c}(\partial V) \ni 1$, applying the fact above, we obtain that there exists $r \in(0,1)$ such that $h_{c}(U) \subset \mathbb{D}_{r}$ and $\log 1 / r>m / 3-M_{0}$. Provided that $m$ is large enough, $r<R$ and $m / 3-\log R / r$ is uniformly bounded from above by some constant $M_{1}$.

Now let us define a new holomorphic motion $\tilde{\mathbf{h}}: D \times V \rightarrow D \times \mathbb{C}$ which coincides with $\mathbf{h}$ on $D \times(\partial V \cup U)$ and such that $\tilde{\mathbf{h}}(c, z)=z$ for all $z \in A(0 ; r, R)$ and $c \in D$. Then $\varphi$ is also a diagonal of this new motion, and $\tilde{\chi}: c \mapsto \tilde{h}_{c}^{-1}(\varphi(c))$ defines a homeomorphism from $D$ onto $V$. Since $\tilde{\chi}^{-1}$ is conformal on the round annulus $A(0 ; r, R)$ and $\tilde{\chi}\left(D^{\prime}\right)=U \subset \mathbb{D}_{r}$, we obtain that

$$
\bmod \left(D \backslash \overline{D^{\prime}}\right) \geqslant \bmod (A(0 ; r, R)) \geqslant \frac{m}{3}-M_{1},
$$

and taking $M=\max \left(M_{0}, M_{1}\right)$, the lemma follows.

\subsection{Parapuzzle}

Let us now define the Yoccoz parapuzzle. For our purpose, it is enough to restrict ourselves to the wake $\mathcal{W}$. Let $\mathcal{X}^{n}=\left\{c \in \mathbb{C} \backslash \mathcal{M}|\log | \Phi(c) \mid<1 / \ell^{n}\right\}$ and $T_{n}=\left\{t \in \mathbb{R} / \mathbb{Z} \mid \ell^{n} t \in\left\{t^{+}, t^{-}\right\}\right\}$. Define

$$
\mathcal{S}^{n}=\partial \mathcal{X}^{n} \cup\left(\bigcup_{t \in T_{n}} \overline{\mathcal{R}(t)}\right) .
$$

A component of $\mathcal{X}^{n} \backslash \mathcal{S}^{n}$ is called a parapuzzle of depth $n$ and denoted by $\mathcal{P}_{n}(c)$ if it contains $c$.

The following lemma describes how the combinatorics of Yoccoz puzzle changes with the parameter.

LEMMA 10 . - Let $c_{0} \in \mathcal{F}_{r}^{0}$. Then for any $n \geqslant 2$, there exists a holomorphic motion

$$
\mathbf{p}_{n}: \mathcal{P}_{n}\left(c_{0}\right) \times \mathbb{C} \rightarrow \mathcal{P}_{n}\left(c_{0}\right) \times \mathbb{C}, \quad(c, z) \mapsto\left(c, p_{n, c}(z)\right)
$$

such that for each $c \in \mathcal{P}_{n}\left(c_{0}\right)$, the following hold:

(1) for each $0 \leqslant i \leqslant n, S_{c}^{i}=p_{n, c}\left(S_{c_{0}}^{i}\right)$;

(2) for each $z \notin X_{c}^{n}, p_{n, c}(z)=B_{c}^{-1} \circ B_{c_{0}}(z)$;

(3) for all $1 \leqslant i \leqslant n$ and all $z \in S_{c_{0}}^{i}, f_{c} \circ p_{n, c}(z)=p_{n, c} \circ f_{c_{0}}(z)$.

Moreover, the restriction $\mathbf{p}_{n} \mid \mathcal{P}_{n}\left(c_{0}\right) \times \overline{P_{c_{0}}^{n}\left(c_{0}\right)}$ is proper filled tube which has the identity map as a diagonal.

Sketch of proof. - We shall only give a sketch of proof here. For the details we refer to Section 2 in [33]. Although only quadratic polynomials are considered there, the proof works through in the general unicritical case.

We take $\mathbf{p}_{n}$ to be the restriction of holomorphic motion $H_{n-1}$ constructed in Lemma 2.5 of [33] to $\mathcal{P}_{n}\left(c_{0}\right) \times \mathbb{C}$. Assuming $n \geqslant 2$, let us show that $\mathbf{p}_{n} \mid \mathcal{P}_{n}\left(c_{0}\right) \times \overline{P_{c_{0}}^{n}\left(c_{0}\right)}$ is a proper tube. For $n=2$, by Lemma 7, we have $P_{c}^{2}(c) \Subset P_{c}^{1}(c)$, which implies that $\mathcal{P}_{2}\left(c_{0}\right) \Subset \mathcal{P}_{1}\left(c_{0}\right)$ by Lemma 2.8 in [33]. For $n>2$ one proceeds by induction. The fact that the identity map is a diagonal to the filled tube follows from Lemma 2.6 in [33]. 
Remark 5. - Clearly, the map $p_{n, c}$ is holomorphic in the region $\left\{z \in \mathbb{C}: G_{c}(z)>1 / \ell^{n}\right\}$. For any $z \in S_{c_{0}}^{n} \backslash K_{c_{0}}, p_{n, c}(z) \in S_{n}^{c} \backslash K_{c}$ and $B_{c} \circ p_{n, c}(z)=B_{c_{0}}(z)$.

Remark 6. - As $t^{+}=-t^{-} \bmod 1$, the set $\mathcal{S}^{n}$ is real-symmetric. Consequently, any parapuzzle piece which intersects $\mathbb{R}$ is real-symmetric.

\section{Properties of the Julia sets}

Given a topological disk $\Omega \subset \mathbb{C}$ and a set $A$, define

$$
\lambda(A \mid \Omega)=\sup _{\varphi} \frac{m(\varphi(A \cap \Omega))}{m(\varphi(\Omega))}
$$

where $\varphi$ runs over all conformal maps from $\Omega$ into $\mathbb{C}$ and $m$ denotes the planar Lebesgue measure.

Definition 5. - Let $V \subset \mathbb{C}$ be a topological disk. Let $U_{i}, i=0,1, \ldots$, be pairwise disjoint topological disks contained in $V$. We say that the family $\left\{U_{i}\right\}$ is $\varepsilon$-absolutely-small in $V$ if $\lambda\left(\bigcup_{i} U_{i} \mid V\right)<\varepsilon$, and for each $i$, the diameter of $U_{i}$ in the hyperbolic metric of $V$ is less than $\varepsilon$. Here the hyperbolic metric of $V$ is the pullback of the standard Poincare metric on the unit disk $\mathbb{D} \subset \mathbb{C}$ by the Riemann mapping from $V$ to $\mathbb{D}$.

The main result of this section is the following:

THEOREM 6. - Consider a map $f=f_{c}$ with $c \in \mathcal{D G}$. Then for any $\varepsilon>0$, there exists a critical puzzle piece $Y$ such that the collection of the components of the domain of the first return map to $Y$ is $\varepsilon$-absolutely-small in $Y$.

\subsection{Extendibility}

For a puzzle piece $Y$, let $D(Y)$ denote the set of all points $z$ for which there exist $k=k(z) \geqslant 1$ with $f^{k}(z) \in Y$, let $E(Y)=D(Y) \cup Y$, and let $g_{Y}: D(Y) \cap Y \rightarrow Y$ denote the first return map to $Y$.

We shall say that a Jordan disk $\widehat{Y} \supset Y$ in $\mathbb{C}$ is an extension domain of $g_{Y}$, if for each component $U$ of $D(Y) \cap Y$, there exists a Jordan disk $\widehat{U}$ with $Y \supset \widehat{U} \supset U$ such that $f^{s-1}: f(\widehat{U}) \rightarrow \widehat{Y}$ is a conformal map, where $s$ denotes the return time of $U$ to $V$, i.e., $g_{V}\left|U=f^{s}\right| U$. We say that $g_{Y}$ is $C$-extendible if there exists an extension domain $\widehat{Y}$ with $\bmod (\widehat{Y} \backslash \bar{Y}) \geqslant C$.

A critical puzzle $Y$ is called $C$-nice if for each return domain $U$ to $Y$ we have $\bmod (Y \backslash \bar{U}) \geqslant C$. Remark that if $g_{Y}$ is $C$-extendible, then $Y$ is $C / \ell$-nice:

$$
\bmod (Y \backslash \bar{U}) \geqslant \bmod (\widehat{U} \backslash \bar{U}) \geqslant \bmod (\widehat{Y} \backslash \bar{Y}) / \ell \geqslant C / \ell .
$$

The following lemma will be convenient for us to find extension domains.

LEMMA 11. - Let $\widehat{Y} \supset Y$ be puzzle pieces such that $f^{k}(\partial Y) \cap \widehat{Y}=\emptyset$ for all $k \geqslant 1$.

- If $Y \ni 0$, then $\widehat{Y}$ is an extension domain of $g_{Y}$.

- If $\widehat{Z}$ is a critical puzzle piece such that $f^{s-1}: f(\widehat{Z}) \rightarrow \widehat{Y}$ is a conformal map for some $s \in \mathbb{N}$, and $f^{s}(0) \in Y$, then $\widehat{Z}$ is an extension domain of $g_{Z}$, where $Z=\operatorname{Comp}_{0}\left(f^{-s}(Y)\right)$.

$4^{\text {e }}$ SÉRIE - TOME $39-2006-\mathrm{N}^{\circ} 3$ 
Proof. - Let $U$ be a return domain to $Y$ and let $r$ be the return time. For each $0 \leqslant i \leqslant r$ let $Q_{i}$ denote the component of $f^{i-r}(\widehat{Y})$ which contains $f^{i}(U)$. For each $0 \leqslant i<r, Q_{i} \cap \partial Y=\emptyset$ for otherwise there exists $z \in \partial Y$ with $f^{r-i}(z) \in \widehat{Y}$. This shows that $Q_{i} \subset Y$ if $0 \in Q_{i}$. In particular, $Q_{0} \subset Y$. Moreover, this implies that $Q_{i} \not \ngtr 0$ for all $0<i<r$. In fact, otherwise, we would have $f^{i}(U) \subset Q_{i} \subset Y$, contradicting the fact that $r$ is the return time of $U$ to $Y$. This proves that $\widehat{Y}$ is an extension domain of $g_{Y}$. For the second statement, one checks that $f^{k}(\partial Z) \cap \widehat{Z}=\emptyset$ for all $k \geqslant 1$ and then applies the first statement of the lemma.

\subsection{A recursive argument}

To prove Theorem 6 let us start with a slightly more general situation.

LEMMA 12. - For any $\varepsilon>0$ there exists $C>0$ such that if $Y$ is a critical puzzle piece and if the first return map $g_{Y}$ is $C$-extendible, then

$$
1-\lambda\left(E\left(Y^{1}\right) \mid Y\right) \geqslant \frac{m(Y \backslash D(Y))}{m(Y \backslash D(Y))+\varepsilon m(Y)}\left(1-\frac{\varepsilon}{4}\right),
$$

where $Y^{1}$ is the critical return domain to $Y$. Moreover, if $Y^{\prime}$ is a child of $Y$, then

$$
1-\lambda\left(D\left(Y^{\prime}\right) \mid Y^{\prime}\right) \geqslant \frac{(1-\varepsilon) m(Y \backslash D(Y))}{m(Y \backslash D(Y))+\varepsilon m(Y)} \geqslant \frac{1-\lambda(D(Y) \mid Y)}{1-\lambda(D(Y) \mid Y)+\varepsilon}(1-\varepsilon) .
$$

Proof. - Let us use $B_{Y}(r)$ to denote the hyperbolic ball in $Y$ with center 0 and radius $r$. Let $\delta>0$ be a small constant so that

$$
\lambda\left(B_{Y}(2 \delta) \mid Y\right) \leqslant \frac{\varepsilon}{4} .
$$

Define $U_{0}=Y \backslash D(Y)$, define $V_{0}$ to be the union of all components $P$ of $Y \cap D(Y)$ with $P \cap B_{Y}(\delta)=\emptyset$, and define $W_{0}$ to be the union of all other component of $D(Y) \cap Y$. Moreover inductively define $U_{i}, V_{i}, W_{i}$ for all $i \geqslant 1$ as follows:

$$
\begin{aligned}
U_{i} & =\left\{z \in V_{i-1} \mid g_{Y}^{i}(z) \in U_{0}\right\} ; \\
V_{i} & =\left\{z \in V_{i-1} \mid g_{Y}^{i}(z) \in V_{0}\right\} ; \\
W_{i} & =\left\{z \in V_{i-1} \mid g_{Y}^{i}(z) \in W_{0}\right\} .
\end{aligned}
$$

By definition of $C$-extendibility, there exits a topological disk $\widehat{Y} \supset Y$ with $\bmod (\widehat{Y} \backslash \bar{Y}) \geqslant C$ and satisfying the following: for each component $P$ of $D(Y) \cap Y$, there exists a topological disk $\widehat{P}$ with $P \subset \widehat{P} \subset Y$ and such that $f^{s-1}: f \widehat{P} \rightarrow \widehat{Y}$ is a conformal map, where $s$ denotes the return time of $P$ into $Y$. Take $\gamma$ to be the core-curve of the annulus $\widehat{Y} \backslash \bar{Y}$, i.e., $\gamma$ is the Jordan curve in $\widehat{Y} \backslash \bar{Y}$ which separate $\widehat{Y} \backslash \bar{Y}$ into two annuli with $\operatorname{modulus} \bmod (\widehat{Y} \backslash \bar{Y}) / 2$. Let $\widetilde{Y}$ be the domain bounded by $\gamma$ and define $\widetilde{P}=\operatorname{Comp}_{P}\left(f^{-s}(\tilde{Y})\right)$. Then $\bmod (Y \backslash \overline{\widetilde{P}}) \geqslant \bmod (\widehat{Y} \backslash \overline{\widetilde{Y}}) /(2 \ell) \geqslant$ $C / 2 \ell$. If $C$ is sufficiently large, then this implies that if $P \subset V_{0}$ then $0 \notin \widetilde{P}$. It follows that for any $i \geqslant 1$ and any component $A$ of $V_{i-1}, R_{Y}^{i} \mid A$ extends to a conformal map onto $\tilde{Y}$. By the Koebe distortion theorem, the distortion $\operatorname{Dist}\left(R_{Y}^{i} \mid A\right)$ is small. Note also that $W_{0} \subset B_{Y}(2 \delta)$. Thus

$$
\frac{m\left(A \cap U_{i}\right)}{m\left(A \cap W_{i}\right)} \geqslant \frac{1}{2} \frac{m\left(U_{0}\right)}{m\left(W_{0}\right)}=\frac{1}{2} \frac{m\left(U_{0}\right)}{m(Y)} \frac{m(Y)}{m\left(W_{0}\right)} \geqslant \frac{2}{\varepsilon} \frac{m\left(U_{0}\right)}{m(Y)} .
$$


Since $E\left(Y^{1}\right) \cap Y \subset \bigcup_{i} W_{i}$, this implies that for each component $P$ of $V_{0}$,

$$
\frac{m\left(P \backslash E\left(Y^{1}\right)\right)}{m\left(P \cap E\left(Y^{1}\right)\right)} \geqslant \frac{2}{\varepsilon} \frac{m\left(U_{0}\right)}{m(Y)} .
$$

Let us estimate $\lambda\left(Y \backslash E\left(Y^{1}\right) \mid Y\right)$. Let $\varphi$ be a conformal map from $Y$ into $\mathbb{C}$. Then

$$
\frac{m\left(\varphi\left(Y \backslash E\left(Y^{1}\right)\right)\right)}{m(\varphi Y)} \geqslant \frac{m\left(\varphi U_{0}\right)}{m(\varphi Y)}+\sum_{P \in \mathcal{V}_{0}} \frac{m\left(\varphi\left(P \backslash E\left(Y^{1}\right)\right)\right)}{m(\varphi(P))} \frac{m(\varphi(P))}{m(\varphi Y)},
$$

where $\mathcal{V}_{0}$ denote the collection of the components of $V_{0}$. As $\bmod (Y \backslash \bar{P}) \geqslant C / \ell$, $\operatorname{Dist}(\varphi \mid P) \ll 1$ provided that $C$ is sufficiently large. By (10), this implies

$$
\frac{m\left(\varphi\left(P \backslash E\left(Y^{1}\right)\right)\right)}{m(\varphi(P))} \geqslant \frac{m\left(U_{0}\right)}{m\left(U_{0}\right)+\varepsilon m(Y)}
$$

and hence

$$
\begin{aligned}
\frac{m\left(\varphi\left(Y \backslash E\left(Y^{1}\right)\right)\right)}{m(\varphi Y)} & \geqslant \frac{m\left(U_{0}\right)}{m\left(U_{0}\right)+\varepsilon m(Y)}\left(\frac{m\left(\varphi U_{0}\right)}{m(\varphi Y)}+\sum_{P \in \mathcal{V}_{0}} \frac{m(\varphi(P))}{m(\varphi Y)}\right) \\
& =\frac{m\left(U_{0}\right)}{m\left(U_{0}\right)+\varepsilon m(Y)}\left(1-\frac{m\left(\varphi\left(W_{0}\right)\right)}{m(\varphi(Y))}\right) \\
& \geqslant \frac{m\left(U_{0}\right)}{m\left(U_{0}\right)+\varepsilon m(Y)}\left(1-\lambda\left(B_{Y}(2 \delta) \mid Y\right)\right) \\
& \geqslant \frac{m\left(U_{0}\right)}{m\left(U_{0}\right)+\varepsilon m(Y)}\left(1-\frac{\varepsilon}{4}\right) .
\end{aligned}
$$

This proves (8).

Now let $Y^{\prime}$ be a child of $Y$ and let $s$ be such that $f^{s}\left(Y^{\prime}\right)=Y$. As $Y^{\prime} \subset Y^{1}$, we have $\lambda\left(E\left(Y^{\prime}\right) \mid Y\right) \leqslant \lambda\left(E\left(Y^{1}\right) \mid Y\right)$. Let $Q_{0} \ni 0, Q_{1}, Q_{2}, \ldots$ be the components of $f^{-s}(D(Y)) \cap Y^{\prime}$, and let $\mathcal{I}=\left\{i \geqslant 0 \mid i=0\right.$ or $\left.f^{s}\left(Q_{i}\right)=Y^{1}\right\}$. Then

$$
\# \mathcal{I} \leqslant \ell+1
$$

As $\bmod \left(Y^{\prime} \backslash \overline{Q_{i}}\right) \geqslant \bmod \left(Y \backslash \overline{f^{s}\left(Q_{i}\right)}\right) / \ell \geqslant C / \ell^{2}$ for all $i$, it follows that

$$
\lambda\left(\bigcup_{i \in \mathcal{I}} Q_{i} \mid Y^{\prime}\right) \leqslant \frac{\varepsilon}{2}
$$

provided that $C$ is sufficiently large. Let $\varphi$ be any conformal map into $\mathbb{C}$, and let $U_{0}^{\prime}=$ $f^{-s}\left(U_{0}\right) \cap Y^{\prime}$. For any $i \notin \mathcal{I}, R_{Y} \circ f^{s}$ maps $Q_{i}$ conformally onto $Y$ and maps $Q_{i} \cap D\left(Y^{\prime}\right)$ onto $Y \cap E\left(Y^{\prime}\right)$, so

$$
\begin{aligned}
\frac{m\left(\varphi\left(Q_{i} \backslash D\left(Y^{\prime}\right)\right)\right)}{m\left(\varphi\left(Q_{i}\right)\right)} & \geqslant 1-\lambda\left(E\left(Y^{\prime}\right) \mid Y\right) \\
& \geqslant \frac{m\left(U_{0}\right)}{m\left(U_{0}\right)+\varepsilon m(Y)}\left(1-\frac{\varepsilon}{4}\right) .
\end{aligned}
$$

$4^{\mathrm{e}}$ SÉRIE - TOME $39-2006-\mathrm{N}^{\circ} 3$ 
Thus

$$
\begin{aligned}
\frac{m\left(\varphi\left(Y^{\prime} \backslash D\left(Y^{\prime}\right)\right)\right)}{m\left(\varphi\left(Y^{\prime}\right)\right)} & =\frac{m\left(\varphi\left(U_{0}^{\prime}\right)\right)}{m\left(\varphi\left(Y^{\prime}\right)\right)}+\sum_{i \notin \mathcal{I}} \frac{m\left(\varphi\left(Q_{i} \backslash D\left(Y^{\prime}\right)\right)\right)}{m\left(\varphi\left(Q_{i}\right)\right)} \frac{m\left(\varphi\left(Q_{i}\right)\right)}{m\left(\varphi\left(Y^{\prime}\right)\right)} \\
& \geqslant \frac{m\left(U_{0}\right)}{m\left(U_{0}\right)+\varepsilon m(Y)}\left(1-\frac{\varepsilon}{4}\right)\left(1-\sum_{i \in \mathcal{I}} \frac{m\left(\varphi\left(Q_{i}\right)\right)}{m\left(\varphi\left(Y^{\prime}\right)\right)}\right) \\
& \geqslant \frac{m\left(U_{0}\right)}{m\left(U_{0}\right)+\varepsilon m(Y)}\left(1-\frac{\varepsilon}{4}\right)\left(1-\frac{\varepsilon}{2}\right) \\
& \geqslant \frac{m\left(U_{0}\right)}{m\left(U_{0}\right)+\varepsilon m(Y)}(1-\varepsilon) \\
& \geqslant \frac{1-\lambda(D(Y) \mid Y)}{1-\lambda(D(Y) \mid Y)+\varepsilon}(1-\varepsilon) .
\end{aligned}
$$

Remark 7. - Note that the first part of (9) implies that (provided that $g_{Y}$ is $C$-extendible with a large $C), 1-\lambda\left(D\left(Y^{\prime}\right) \mid Y^{\prime}\right)>0$. This follows from the simple observation that $Y-D(Y)$ has a non-empty interior.

\subsection{Proof of Theorem 6}

PROPOSITION 4. - Assume that $c \in \mathcal{D} \mathcal{G}$. Then there exists a sequence of critical puzzle pieces

$$
Y_{1} \ni Y_{2} \ni Y_{3} \ni \cdots
$$

and a sequence of numbers $C_{n} \rightarrow \infty$ as $n \rightarrow \infty$ such that the following hold:

- for each $n, Y_{n+1}$ is a child of $Y_{n}$;

- the first return map to $Y_{n}$ is $C_{n}$-extendible.

Proof. - In the proof we will drop the parameter $c$ from the notation. We shall distinguish two cases.

CASE $1 .-f_{c}$ is reluctantly recurrent.

Step 1. Let $N \in \mathbb{N}$ be such that $P^{N}(0)$ has infinitely many children. Then for all $n \geqslant N, P^{n}(0)$ has infinitely many children. In fact, if $P^{N+s}(0)$ is a child of $P^{N}(0)$, and if $k \geqslant 0$ is minimal such that $f^{s+k}(0) \in P^{n}(0)$, then $P^{n+k+s}(0)$ is a child of $P^{n}(0)$.

Step 2. Let $V$ be a critical puzzle piece of depth $\geqslant N$, and let $U$ be its central return domain. We claim that there exists an arbitrarily large $s \in \mathbb{N}$, such that $f^{s}(0) \in U$ and $W=\operatorname{Comp}_{0}\left(f^{-s}(V)\right)$ is a child of $V$.

To see this, fix a positive integer $M$. There exists $s_{1}>M$ such that $W_{1}=\operatorname{Comp}_{0}\left(f^{-s_{1}}(V)\right)$ is a child of $V$. As 0 is recurrent, there exists a minimal $m \in \mathbb{N} \cup\{0\}$ such that $g_{V}^{m}\left(f^{s_{1}}(0)\right) \in U$, where $g_{V}$ denotes the first return map to $V$. By minimality of $m$, there exists a neighborhood $Q$ of $f^{s_{1}}(0)$ such that $g_{V}^{m}$ maps $Q$ conformally onto $V$. Let $W:=\operatorname{Comp}_{0}\left(f^{-s_{1}}(Q)\right)$. Then clearly $W$ is a child of $V$ with transition time $s \geqslant s_{1}>M$ (i.e., $\left.W=\operatorname{Comp}_{0}\left(f^{-s}(V)\right)\right)$ and $f^{s}(0) \in U$.

Step 3. Let $U, V$ be as in step 2. Assume that $U \Subset V$. Let us show that for every $C>0$, any child $W$ of $V$ with a sufficiently large transition time is $C$-nice.

Let $s_{1}<s_{2}<\cdots$ be all the positive integers such that $f^{s_{n}}(0) \in U$ and such that $W_{n}^{\prime}=$ $\operatorname{Comp}_{0}\left(f^{-s_{n}}(V)\right)$ is a child of $V, n \geqslant 1$. Then $W_{n}:=\operatorname{Comp}_{0}\left(f^{-s_{n}}(U)\right)$ is a child of $U$. Let $W_{0}^{\prime}=V$ and $W_{0}=U$. Note that $W_{n} \supset W_{n+1}^{\prime}$ for all $n$ (because otherwise, as these puzzle pieces both contain the critical point, $W_{n+1}^{\prime} \supset W_{n}$; this would imply that $f^{s_{n}}\left(W_{n+1}^{\prime}\right) \supset U \ni 0$, which is impossible since $W_{n+1}^{\prime}=\operatorname{Comp}_{0}\left(f^{-s_{n+1}}(V)\right)$ is a child of $V$ and therefore $f^{s_{n+1}-1} \mid f\left(W_{n+1}^{\prime}\right)$ 
is univalent). For all $n \geqslant 1$, since $f^{s_{n}}: W_{n}^{\prime} \backslash \bar{W}_{n} \rightarrow V \backslash \bar{U}$ is a covering map of degree $\ell$,

$$
\bmod \left(W_{n}^{\prime} \backslash \bar{W}_{n}\right)=\mu:=\bmod (V \backslash \bar{U}) / \ell>0 .
$$

To complete this step, let us show that if $W$ is a child of $V$ such that $W \subset W_{n-1}$, then $W$ is $n \mu / \ell$-nice.

To this end, let $s \in \mathbb{N}$ be such that $f^{s}(W)=V$. Let $P$ be a return domain to $W$ and let $r$ be the return time. Clearly, $r \geqslant s$. If $r=s$, then $f^{s}(P)=W$, so

$$
\bmod (W \backslash \bar{P}) \geqslant \frac{\bmod (V \backslash \bar{W})}{\ell} \geqslant \ell^{-1} \sum_{i=0}^{n-1} \bmod \left(W_{i}^{\prime} \backslash \bar{W}_{i}\right) \geqslant n \mu / \ell
$$

If $r>s$, then $f^{s}(P)$ is a landing domain to $W$. For $0 \leqslant i \leqslant n-1$, let $Q_{i}^{\prime}, Q_{i} \supset f^{s}(P)$ denote the landing domain to $W_{i}^{\prime}$ and $W_{i}$ respectively. Then $\bmod \left(Q_{i}^{\prime} \backslash \overline{Q_{i}}\right) \geqslant \bmod \left(W_{i}^{\prime} \backslash \overline{W_{i}}\right) \geqslant \mu$. Since $f^{s}(P) \subset Q_{n-1}$, it follows that $\bmod \left(V \backslash \overline{f^{s}(P)}\right) \geqslant n \mu$ and hence

$$
\bmod (W \backslash \bar{P}) \geqslant \bmod \left(V \backslash \overline{f^{s}(P)}\right) / \ell \geqslant n \mu / \ell .
$$

Step 4. Let us now complete the proof of Proposition 4 in the reluctantly recurrent case.

Let us first prove that there exists a 1-nice critical puzzle piece $Y_{1}$. Take a critical puzzle piece $V$ of depth $\geqslant N$, such that its central return domain $U$ is compactly contained in $V$. Such a puzzle piece exists: one can take $V$ to be a critical pull back of $P^{3}(0)$. By step 3, $V$ has a 1-nice child which is $Y_{1}$.

Once $Y_{2 n-1}$ is defined, let $Y_{2 n}$ its the central return domain. By steps 2 and 3, there exists $s_{n} \in \mathbb{N}$, such that $f^{s_{n}}(0) \in Y_{2 n}$, and $W_{n}^{\prime}=\operatorname{Comp}_{0}\left(f^{-s_{n}}\left(Y_{2 n-1}\right)\right)$ is a child of $Y_{2 n-1}$ and $W_{n}=\operatorname{Comp}_{0}\left(f^{-s_{n}}\left(Y_{2 n}\right)\right)$ is $(n+1)$-nice a child of $Y_{2 n}$. Define $Y^{2 n+1}=W_{n}$. Note that by Lemma $11, W_{n}^{\prime}$ is an extension domain of the first return map to $Y_{2 n+1}$. It is easy to see that so defined $Y_{n}, n \geqslant 1$ satisfies all the requirement in this proposition.

CASE 2. $-f_{c}$ is persistently recurrent and there exists a chain of nice intervals $\Gamma^{0} \supset \Gamma^{1} \supset$ $\cdots \ni 0$ such that $\Gamma^{n+1}$ is the smallest child of $\Gamma^{n}$ and so that $\left|\Gamma^{n+1}\right| /\left|\Gamma^{n}\right| \rightarrow 0$ as $n \rightarrow \infty$. Now let us consider the enhanced nest of puzzle pieces $\mathbf{I}_{n} \supset \mathbf{L}_{n} \supset \mathbf{K}_{n} \supset \mathbf{I}_{n+1} \supset \cdots$ defined in Section 8 of [19] and let $I_{n}, L_{n}, K_{n}$ be their real traces. This construction is based on the fact that to each critical puzzle piece $\mathbf{I}$ one can associate a positive integer $\nu$ so that $f^{\nu}(0) \in \mathbf{I}$ and if we define

$$
\mathcal{A}(\mathbf{I}):=\operatorname{Comp}_{0}\left(f^{-\nu}\left(\mathcal{L}_{f^{\nu}(0)}(\mathbf{I})\right)\right) \subset \mathcal{B}(\mathbf{I}):=\operatorname{Comp}_{0}\left(f^{-\nu}(\mathbf{I})\right)
$$

then $f^{\nu}: \mathcal{B}(\mathbf{I}) \rightarrow \mathbf{I}$ has degree bounded by some universal constant and $\mathcal{B}(\mathbf{I})-\mathcal{A}(\mathbf{I})$ is disjoint from the orbit of the critical point. (In fact, in the unicritical case one can choose $\nu$ so that $\mathcal{L}_{f^{\nu}(0)}(\mathbf{I})=\mathcal{L}_{0}(\mathbf{I})$.) If we denote the smallest child of $\mathbf{I}$ by $\Gamma(\mathbf{I})$ then the enhanced nest is inductively defined by $\mathbf{L}_{n}=\mathcal{A}\left(\mathbf{I}_{n}\right), \mathbf{K}_{n}=\mathcal{B}\left(\mathbf{L}_{n}\right), \mathbf{I}_{n+1}=\Gamma^{T}\left(\mathbf{K}_{n}\right)$ where $T$ is a fixed integer chosen in Section 8.1 of [19]. By this construction, there exist integers $s_{n}, t_{n}, q_{n}$ so that $\mathbf{L}_{n}=\operatorname{Comp}_{0}\left(f^{-s_{n}}\left(\mathbf{I}_{n}\right)\right), \mathbf{K}_{n}=\operatorname{Comp}_{0}\left(f^{-t_{n}}\left(\mathbf{L}_{n}\right)\right)$ and $\mathbf{I}_{n+1}=\operatorname{Comp}_{0}\left(f^{-q_{n}}\left(\mathbf{K}_{n}\right)\right)$, i.e., $f^{s_{n}+t_{n}+q_{n}}\left(\mathbf{I}_{n+1}\right)=\mathbf{I}_{n}$ and, moreover, there exists some fixed $T^{\prime}$ so that for each $n$ at most $T^{\prime}$ of the iterates $f^{i}\left(\mathbf{I}_{n+1}\right), i=0, \ldots, s_{n}+t_{n}+q_{n}$, contains the critical point. So although $\mathbf{I}_{n+1}, \mathbf{L}_{n}, \mathbf{K}_{n}$ may not be children of $\mathbf{I}_{n}$, they are descendants (i.e. a child, or a child of a child and so on) of $\mathbf{I}_{n}$ of generation $\leqslant T^{\prime}$. In other words, by picking all puzzle pieces in this pullback which contain the critical point, we obtain the sequence $Y_{0} \ni Y_{1} \ni Y_{2} \ni \cdots$ such 
that for each $n, Y_{n+1}$ is a child of $Y_{n}$ and so that the puzzle pieces from the enhanced nest $\mathbf{I}_{n} \supset \mathbf{L}_{n} \supset \mathbf{K}_{n} \supset \mathbf{I}_{n+1} \supset \cdots$ all appear in the sequence $Y_{1}, Y_{2}, \ldots$ (and so the integers $u_{n}$ defined by $Y_{u_{n}}=\mathbf{I}_{n}$ satisfy $u_{n+1} \leqslant u_{n}+T^{\prime}$ ). By the Key Lemma stated in Section 4 in [19], there exists $\eta=\eta(\ell)>0$ such that for all $n$ sufficiently large, $\mathbf{I}_{n}$ has $\eta$-bounded geometry: $B\left(0, \eta \operatorname{diam}\left(\mathbf{I}_{n}\right)\right) \subset \mathbf{I}_{n}$. Moreover, there exists $\xi>0$ and a neighborhood $\mathbf{I}_{n}^{\prime}$ of $\mathbf{I}_{n}$ so that $\mathbf{I}_{n}^{\prime} \cap \omega(0) \subset \mathbf{I}_{n}$ and $\bmod \left(\mathbf{I}_{n}^{\prime} \backslash \mathbf{I}_{n}\right) \geqslant \xi$ for each $n \geqslant 0$. It follows that all $Y_{i}$ have $\eta^{\prime}$ bounded geometry for all $i$ large, see [19].

By construction, for any $n$, there are at least two nice intervals $\Gamma^{i}$ and $\Gamma^{i+1}$ between $I_{n}$ and $I_{n+1}$. It follows that $\left|I_{n+1}\right| /\left|I_{n}\right|$ tends to zero. Hence, by part 3 of Proposition 8.1 in [19] (and the definition above that proposition) there exists $\rho_{n} \rightarrow \infty$ so that (using the notation from the end of Section 1),

$$
\left(1+2 \rho_{n}\right) \mathcal{L}_{x}\left(I_{n}\right) \subset I_{n} \quad \text { for all } x \in \omega(0) \cap I_{n},
$$

and

$$
\left(\left(1+2 \rho_{n}\right) I_{n}-\left(1+2 \rho_{n}\right)^{-1} I_{n}\right) \cap \omega(0)=\emptyset .
$$

Hence $\bmod \left(\mathbf{I}_{n}-\mathcal{L}_{f^{\nu_{n}(0)}}\left(\mathbf{I}_{n}\right)\right) \rightarrow \infty$. Since $f^{k}\left(\partial \mathcal{L}_{f^{\nu_{n}(0)}}\left(\mathbf{I}_{n}\right)\right) \cap \operatorname{int}\left(\mathbf{I}_{n}\right)=\emptyset$ for all $k \geqslant 1$, we can apply the second part of Lemma 11 (possibly repeatedly if $\mathcal{B}\left(\mathbf{I}_{n}\right)$ is not a child, but a grandchild of $\mathbf{I}_{n}$ ), and obtain that $\mathcal{B}\left(\mathbf{I}_{n}\right)$ is a $C_{n}$-extension domain of the first return map to $\mathbf{L}_{n}=\mathcal{A}\left(\mathbf{I}_{n}\right)$ with $C_{n} \rightarrow \infty$. Since $\mathcal{B}\left(\mathbf{I}_{n}\right) \backslash \mathbf{L}_{n}$ is disjoint from the critical set, we can repeatedly apply the second part of Lemma 11 to the children (and their children) of $\mathbf{L}_{n}$. Since we only need to repeat this at most $T^{\prime}$ times until we get to $\mathbf{L}_{n+1}$, this implies the $C_{i}^{\prime}$-extendibility of the first return maps to each of the puzzle pieces $Y_{i}$ with $C_{i}^{\prime} \rightarrow \infty$.

Proof of Theorem 6. - Let $Y_{n}, n \geqslant 1$, be as in the above proposition, and let $\mu_{n}=$ $1-\lambda\left(D\left(Y_{n}\right) \mid Y_{n}\right)$. By Remark 7, there exists $n_{0}$ such that for all $n \geqslant n_{0}, \mu_{n}>0$. By Lemma 12, for any $\varepsilon>0$,

$$
\mu_{n+1} \geqslant \frac{\mu_{n}}{\mu_{n}+\varepsilon}(1-\varepsilon),
$$

holds for all $n$ sufficiently large, which implies that

$$
\liminf _{n \rightarrow \infty} \mu_{n} \geqslant 1-2 \varepsilon
$$

Therefore, $\lim _{n} \mu_{n}=1$.

\section{Pseudo-conjugacy}

Definition 6. - Let $g: \bigcup_{i} U_{i} \rightarrow V$ and $\tilde{g}: \bigcup_{i} \widetilde{U}_{i} \rightarrow \widetilde{V}$ be R-maps. A qc map $\varphi: \mathbb{C} \rightarrow \mathbb{C}$ is called a pseudo-conjugacy between them if $\varphi$ maps $V$ onto $\widetilde{V}, U_{i}$ onto $\widetilde{U}_{i}$, and respects the boundary dynamics: for each $z \in \partial U_{i}, \varphi \circ g(z)=\tilde{g} \circ \varphi(z)$.

PROPOSITION 5. - There exists a universal constant $\varepsilon_{0}>0$ such that the following holds. Let $g$ and $\tilde{g}$ be R-maps as above, and let $\varphi$ be a pseudo-conjugacy between them which is conformal a.e. in $V \backslash\left(\bigcup U_{i}\right)$. Assume that $\left\{U_{i}\right\}$ is $\varepsilon_{0}$-absolutely-small in $V$. Then there exists a 2-qc map $\theta_{0}: V \rightarrow \widetilde{V}$ which coincides with $\varphi$ on $\partial V$. Moreover, there exists a qc map $\psi: \mathbb{C} \rightarrow \mathbb{C}$ such that $\psi=\varphi$ on $\mathbb{C} \backslash \bigcup_{i} U_{i}$ (thus $\psi$ is a pseudo-conjugacy), and such that $\psi$ is 2 -qc on $V \backslash \overline{U_{0}}$.

For the proof we need the following lemma. 
LEMMA 13. - There exists an $\varepsilon_{1}>0$ with the following property. Let $\varphi: \Omega \rightarrow \widetilde{\Omega}$ be a $K-q c$ map between Jordan disks in $\mathbb{C}$ and let $A \subset \Omega$ be a measurable set with $\lambda(A \mid \Omega) \leqslant \varepsilon_{1}$. Assume that $\varphi$ is conformal a.e. outside $A$. Then there exists a $\max (K / 4,2)-q c$ map $\hat{\varphi}$ such that $\hat{\varphi} \mid \partial \Omega$ agrees with the continuous extension of $\varphi$ to $\partial \Omega$.

Proof. - Without loss of generality we may assume that $\Omega=\widetilde{\Omega}=\mathbb{D}$. Moreover, we may assume that $K \leqslant 8$, because otherwise $\varphi$ can be written as the decomposition of two qc maps $\varphi_{2} \circ \varphi_{1}$, such that $\varphi_{1}$ is 8-qc and conformal a.e. outside $A$, and $\varphi_{2}$ is $K / 8$-qc.

Assuming that $\varepsilon_{1}$ is small, let us prove that $\varphi \mid \partial \mathbb{D}$ extends to a 2-qc map from $\mathbb{D}$ onto itself. By classical quasiconformal mapping theory, it suffices to show that if $a, b, c, d$ are consecutive distinct points in $\partial \mathbb{D}$ with

$$
C r(a, b, c, d):=\frac{d-a}{c-a} \frac{c-b}{d-b}=\frac{1}{2},
$$

then $\operatorname{Cr}(h(a), h(b), h(c), h(d))$ is close to $1 / 2$. Let us consider Möbius transformations $\sigma, \tau$ such that $\sigma(a, b, c)=\tau(h(a), h(b), h(c))=(1,-i,-1)$, and let $\tilde{\varphi}=\tau \circ \varphi \circ \sigma^{-1}$. Notice that $\sigma(d)=-i$ and $\tau(\varphi(d))=\tilde{\varphi}(-i)$. It suffices to show that $\tilde{\varphi}(-i)$ is close to $-i$. Note that $\tilde{\varphi}$ is 8-qc and conformal a.e. outside $\tilde{A}=\sigma(A)$. As

$$
\frac{m(\tilde{A})}{m(\mathbb{D})} \leqslant \lambda(A \mid \mathbb{D})<\varepsilon_{1},
$$

the desired estimate follows from the formula for the solution of Beltrami equations. See Chapter 5 of [1].

Proof of Proposition 5. - Let $\mathcal{Q}$ be the collection of all qc maps $\theta: V \rightarrow \widetilde{V}$ which coincide with $\varphi$ on $\partial V$, and let $K_{0}=\inf \{K(\theta): \theta \in \mathcal{Q}\}$, where $K(\theta)$ denotes the maximal dilatation of $\theta$. For each $K \geqslant 1$, all $K$-qc maps in $\mathcal{Q}$ form a compact family, so there exists $\theta_{0} \in \mathcal{Q}$ which is $K_{0}$-qc.

Define $\psi: V \rightarrow \widetilde{V}$ to be the map such that $\psi=\varphi$ on $V \backslash \bigcup_{i \neq 0} U_{i}$, and such that $\tilde{g} \circ \psi=\theta_{0} \circ g$ holds on $\bigcup_{i \neq 0} U_{i}$. Then $\psi$ is a qc map. In fact, for each $k \in \mathbb{N}$ there exists a homeomorphism $\psi_{k}: V \rightarrow \widetilde{V}$ such that $\psi_{k}=\psi$ in $\bigcup_{i=1}^{k} U_{i}$ and $\psi_{k}=\varphi$ otherwise. By Lemma 2 in [12], for each $k$, $\psi_{k}$ is qc with $K\left(\psi_{k}\right) \leqslant \max \left(K(\varphi), K_{0}\right)$, thus $\psi=\lim _{k} \psi_{k}$ is qc. Note that $\psi$ is conformal a.e. on $V \backslash\left(\bigcup_{i} U_{i}\right)$ because it coincides with $\varphi$ in that region. Moreover, $\psi$ is $K_{0}$-qc on $\bigcup_{i \neq 0} U_{i}$.

Now let us apply Lemma 13 to show that there exists a map $\theta_{1} \in \mathcal{Q}$ which is $\max \left(K_{0} / 2,2\right)$ qc. Let $\gamma \subset V \backslash U_{0}$ be the Jordan curve which separates $V \backslash \bar{U}_{0}$ into two annuli with modulus $\bmod \left(V \backslash \overline{U_{0}}\right) / 2$ and let $A_{0}$ be the Jordan disk bounded by $\gamma$. Then provided that $\varepsilon_{0}<\varepsilon_{1} / 2$ is small enough, $\bmod \left(V \backslash A_{0}\right)$ is large, so that $\lambda\left(A_{0} \mid V\right)<\varepsilon_{1} / 2$. Let $A_{1}=\bigcup_{i \neq 0} U_{i}, A=A_{0} \cup A_{1}$. Then $\lambda(A \mid V)<\varepsilon_{1}$.

Let us prove that there exists a $2 K_{0}$-qc map $\chi: A_{0} \rightarrow \psi\left(A_{0}\right)$ with $\chi=\psi$ on $\partial A_{0}$. In fact, using the Measurable Riemann Mapping theorem, there exists a $K_{0}$-qc map $\psi_{1}$ defined on $\psi\left(A_{0}\right)$ such that $\psi_{1} \circ \psi$ is conformal on $A_{0} \backslash \overline{U_{0}}$. Considering the round annuli model, it is easy to show that there exists a 2-qc map $\chi_{1}: A_{0} \rightarrow \psi\left(A_{0}\right)$ such that $\chi_{1}=\psi_{1} \circ \psi$ on $\partial A_{0}$, provided that $\bmod \left(A_{0} \backslash \overline{U_{0}}\right)$ is large enough. Thus $\chi=\psi_{1}^{-1} \circ \chi_{1}$ satisfies the required property.

Extend $\chi$ to be a qc map from $V$ to $\widetilde{V}$ by setting $\chi=\psi$ on $V \backslash A_{0}$. Then $\chi$ is a $2 K_{0}$-qc map which is conformal a.e. outside $A$. The existence of $\theta_{1}$ is then guaranteed by Lemma 13 .

By the minimality of $K_{0}$, we have $K_{0} \leqslant \max \left(K_{0} / 2,2\right)$, i.e., $K_{0} \leqslant 2$. The map $\psi$ constructed above can be extended in an obvious way to a pseudo-conjugacy satisfying all the requirements.

$4^{\text {e }}$ SÉRIE - TOME $39-2006-\mathrm{N}^{\circ} 3$ 


\section{R-families}

\subsection{Construction of R-families}

To transfer information from the dynamical plane to the parameter plane, we shall use the techniques introduced in $[3,22]$. We shall need the notion of R-family.

Definition 7. - Let $D \subset \mathbb{C}$ be a Jordan disk and let $c_{0} \in D$. An $R$-family over $\left(D, c_{0}\right)$ is a family $g$ of R-maps

$$
g_{c}: \bigcup_{i=0}^{\infty} U_{i, c} \rightarrow V_{c}, \quad c \in D
$$

with the following properties:

- $(c, z) \mapsto\left(c, g_{c}(z)\right)$ is holomorphic in both variables $c$ and $z$;

- there exists a holomorphic motion $\mathbf{h}$ of $\mathbb{C}$ over $\left(D, c_{0}\right)$ such that for each $c \in D, h_{c}$ is a pseudo-conjugacy between $g_{c_{0}}$ and $g_{c}$;

- the filled tube $\mathbf{h} \mid D \times \overline{V_{c_{0}}}$ is proper, and the map $c \mapsto g_{c}(0)$ is a diagonal of this filled tube. We shall say that $\mathbf{h}$ is an equipment of $\mathbf{g}$ and that $(\mathbf{g}, \mathbf{h})$ is an equipped $R$-family.

Let us say that an R-family is well-controlled if for each $c \in D$, there exists a qc map $\psi_{c}: \mathbb{C} \rightarrow \mathbb{C}$ such that $\psi_{c}=h_{c}$ on $\partial V_{c_{0}} \cup\left(\bigcup_{i} \partial U_{i, c_{0}}\right)$ (so $\psi_{c}$ is a pseudo-conjugacy between $g_{c_{0}}$ and $g_{c}$ ), and such that $\psi_{c}$ is 2 -qc on $\mathbb{C} \backslash \overline{U_{0, c_{0}}}$.

The following proposition tells us how to obtain an R-family.

Proposition 6. - Let $c_{0} \in \mathcal{W}$ and let $n \in \mathbb{N}$ be such that for all $k \geqslant 1$ we have $f_{c_{0}}^{k}\left(\partial P_{c_{0}}^{n}(0)\right) \cap \overline{P_{c_{0}}^{n}(0)}=\emptyset$ and such that there exists $s \in \mathbb{N}$ with $f_{c_{0}}^{s}(0) \in P_{c_{0}}^{n}(0)$. Denote by $s_{0}$ the least such $s$. Then for each $c \in \mathcal{P}_{n+s_{0}-1}\left(c_{0}\right)$, the first return map $g_{c}$ to $P_{c}^{n}(0)$ under $f_{c}$ is an R-map, and

$$
g_{c}, \quad c \in \mathcal{P}_{n+s_{0}-1}
$$

is an R-family. Moreover, this family has an equipment

$$
\mathbf{h}: \mathcal{P}_{n+s_{0}-1}\left(c_{0}\right) \times \mathbb{C} \rightarrow \mathcal{P}_{n+s_{0}-1}\left(c_{0}\right) \times \mathbb{C}
$$

such that $\mathbf{h}(c, \cdot)$ is conformal a.e. on $P_{c}^{n}(0) \backslash \operatorname{dom}\left(g_{c_{0}}\right)$. Furthermore, if for each $c \in \mathcal{P}_{n+s_{0}-1}\left(c_{0}\right)$, there exists a 2-qc map $\hat{h}_{c}: P_{c_{0}}^{n}(0) \rightarrow P_{c}^{n}(0)$ which extends homeomorphically to $\hat{h}_{c}: \overline{P_{c_{0}}^{n}(0)} \rightarrow \overline{P_{c}^{n}(0)}$ and coincides with $h_{c}$ on $\partial P_{c_{0}}^{n}(0)$, then the $R$-family is well-controlled.

Proof. - Let $\mathbf{p}_{n}: \mathcal{P}_{n} \times \mathbb{C} \rightarrow \mathcal{P}_{n} \times \mathbb{C}$ be the holomorphic motion as in Lemma 10. Let $Y_{1}, Y_{2}, \ldots, Y_{N}$ be all the off-critical puzzle pieces of depth $n$ for $f_{c_{0}}$, and let $Y_{i, c}=p_{n, c}\left(Y_{i}\right)$. For any word $\mathbf{i}=i_{0} i_{1} \cdots i_{k-1} \in\{1,2, \ldots, N\}^{k}, k \geqslant 1$, denote $|\mathbf{i}|=k$ and define

$$
\begin{aligned}
Y_{\mathbf{i}, c} & =\left\{z \in Y_{i_{0}, c} \mid f_{c}^{j}(z) \in Y_{i_{j}, c}, j=0,1, \ldots, k-1\right\} \\
W_{\mathbf{i}, c} & =\left\{z \in Y_{\mathbf{i}, c} \mid f_{c}^{k}(z) \in P_{c}^{n}(0)\right\} .
\end{aligned}
$$

For distinct words of possibly different length, the corresponding sets $Y_{\mathbf{i}, c}$ are either disjoint or nested, whereas the corresponding $W_{\mathbf{i}, c}$ are pairwise disjoint and disjoint from $P_{c}^{n}(0)$. For each $\mathbf{i} \in\{1,2, \ldots, N\}^{k}$ and each $c \in \mathcal{P}_{n}$, there exists a unique qc map $\varphi_{\mathbf{i}, c}: Y_{\mathbf{i}, c_{0}} \rightarrow Y_{\mathbf{i}, c}$ such that $f_{c}^{k} \circ \varphi_{\mathbf{i}, c}=p_{n, c} \circ f_{c_{0}}^{k}$, which maps $Y_{\mathbf{i} j, c_{0}}$ onto $Y_{\mathbf{i} j, c}$ for every $j \in\{1, \ldots, N\}$ and $W_{\mathbf{i}, c_{0}}$ onto $W_{\mathbf{i}, c}$. It follows from part 2 of Lemma 10 that $\varphi_{\mathbf{i}, c}$ is conformal a.e. on $Y_{\mathbf{i}, c_{0}} \backslash\left(W_{\mathbf{i}, c_{0}} \cup \bigcup_{j=1}^{N} Y_{\mathbf{i} j, c_{0}}\right)$. 
Note that

$$
Q_{c}:=\bigcap_{k} \bigcup_{|\mathbf{i}|=k} Y_{\mathbf{i}, c}=\left\{z \in K_{c}: f^{k}(z) \notin P_{c}^{n}(0) \text { for all } k \geqslant 0\right\},
$$

is a hyperbolic set, and thus has zero measure. Define

$$
\varphi_{c}(z)= \begin{cases}\varphi_{\mathbf{i}, c}(z) & \text { if } z \in Y_{\mathbf{i}, c_{0}} \backslash \bigcup_{j=1}^{N} Y_{\mathbf{i}_{j}, c_{0}}, \\ p_{n, c}(z) & \text { if } G_{c_{0}}(z) \geqslant 1 / \ell^{n} .\end{cases}
$$

Then $\Phi(c, z)=\left(c, \varphi_{c}(z)\right)$ defines a holomorphic motion of the set $\mathbb{C} \backslash Q_{c_{0}}$ over $\mathcal{P}_{n}\left(c_{0}\right)$. By the optimal $\lambda$-lemma, it extends to a holomorphic motion of $\mathbb{C}$ over $\mathcal{P}_{n}\left(c_{0}\right)$, again denoted by $\Phi$. Since $Q_{c_{0}}$ has zero planar measure, $\varphi_{c}: \mathbb{C} \rightarrow \mathbb{C}$ is conformal a.e. outside $\bigcup_{\mathbf{i}} W_{\mathbf{i}, c_{0}}$. Note that for all $0 \leqslant k \leqslant n+s_{0}-1, \varphi_{c}\left|P_{c_{0}}^{k}\left(c_{0}\right)=p_{k, c}\right| P_{c_{0}}^{k}\left(c_{0}\right)$. In particular, the identity map is a diagonal of the filled tube $\Phi \mid \mathcal{P}_{n}\left(c_{0}\right) \times \overline{P_{c_{0}}^{n}\left(c_{0}\right)}$.

Let $\left\{\mathbf{i}_{0}, \mathbf{i}_{1}, \ldots\right\}$ be the set of all words (of arbitrary length) such that $W_{\mathbf{i}_{j}, c_{0}} \subset P_{c_{0}}^{n-1}\left(c_{0}\right)$, so organized that $W_{\mathbf{i}_{0}, c_{0}} \ni c_{0}$. Then the union of all $U_{j, c}:=f_{c}^{-1}\left(W_{\mathbf{i}_{j}, c}\right)$ is the domain of $g_{c}$, and $g_{c}\left|U_{j, c}=f_{c}^{\left|\mathbf{i}_{j}\right|+1}\right| U_{j, c}$. By assumption, for all $j, U_{j, c_{0}} \Subset P_{c_{0}}^{n}(0)$, which implies that $U_{j, c} \Subset P_{c}^{n}(0)$ for all $c \in \mathcal{P}_{n}$.

Clearly, $\mathcal{P}_{n+s_{0}-1}\left(c_{0}\right)=\left\{c \in \mathcal{P}_{n} \mid c \in W_{\mathbf{i}_{0}, c}\right\}$. For $c \in \mathcal{P}_{n+s_{0}-1}$, the first return map $g_{c}$ is an R-map. Finally, define a holomorphic motion $\widetilde{\Phi}(c, z)=(c, \tilde{\varphi}(c, z))$ of $\mathbb{C}$ over $\mathcal{P}_{n+s_{0}-1}$ such that $\tilde{\varphi}(c, z)=\varphi_{c}(z)$ if $z \notin W_{\mathbf{i}_{0}, c_{0}}$ and such that $\tilde{\varphi}\left(c, c_{0}\right)=(c, c)$. By pulling back $\widetilde{\Phi}$ we obtain a holomorphic motion $\mathbf{h}(c, z)=\left(c, h_{c}(z)\right)$ of $\mathbb{C}$ over $\mathcal{P}_{n+s_{0}-1}$ with the desired properties.

Let us prove the last statement. Assume that there exists a 2-qc map $\hat{h}_{c}: P_{c_{0}}^{n}(0) \rightarrow P_{c}^{n}(0)$ which coincides with $h_{c}$ on $\partial P_{c_{0}}^{n}(0)$. Since $h_{c}=p_{n, c}$ on $\partial P_{c_{0}}^{n}(0)$, for each word $\mathbf{i}$, we obtain (by pulling back $\hat{h}_{c}$ ) a 2-qc map $\hat{\varphi}_{\mathbf{i}, c}: W_{\mathbf{i}, c_{0}} \rightarrow W_{\mathbf{i}, c}$ which coincides with $\varphi_{c}$ on $\partial W_{\mathbf{i}, c_{0}}$. It follows that there exists a 2-qc map $\hat{\varphi}_{c}: \mathbb{C} \rightarrow \mathbb{C}$ which agrees with $\varphi_{c}$ outside $\bigcup_{\mathbf{i}} W_{\mathbf{i}, c} \cup P_{c_{0}}^{n}(0)$. Now pullback $\hat{\varphi}_{c}$ once more by the first entry maps to $P_{c_{0}}^{n}(0)$ and $P_{c}^{n}(0)$ (restricted to the domains of these maps); elsewhere we keep $\hat{\varphi}_{c}$ as before. In this way we get a pseudo-conjugacy between $g_{c_{0}}$ and $g_{c}$ which coincides with $h_{c}$ on $\mathbb{C} \backslash \operatorname{dom}\left(g_{c_{0}}\right)$ and which is 2 -qc on $\mathbb{C} \backslash \overline{P_{c_{0}}^{n+s_{0}}(0)}$. The statement follows.

Definition 8. - Let us say that an R-family $\mathbf{g}=\left\{g_{c}: \bigcup_{j=0}^{\infty} U_{c}^{j} \rightarrow V_{c}\right\}$ is standard if it can be obtained as in the proposition. Thus any standard R-family is based over a parapuzzle piece $\mathcal{P}_{m}\left(c_{0}\right)$, and it has an equipment $\mathbf{h}$ so that $h_{c}$ is conformal a.e. in $V_{c_{0}} \backslash \operatorname{dom}\left(g_{c_{0}}\right)$.

\subsection{Renormalization of R-families}

Let $D \subset \mathbb{C}$ be a Jordan disk, and let us consider an R-family

$$
\mathbf{g}=\left\{g_{c} \mid \bigcup_{i} U_{i, c} \rightarrow V_{c}, c \in D\right\} .
$$

We shall use holomorphic motion to relate some sets in the dynamical plane with some sets in the parameter plane. More precisely, for each word $\mathbf{i}=i_{0} i_{1} \cdots i_{k-1}$ of non-zero integers define

$$
\begin{aligned}
& D_{\mathbf{i}}=\left\{c \in D \mid g_{c}^{j}\left(g_{c}(0)\right) \in U_{i_{j}, c} \text { for } j=0,1, \ldots, k-1\right\} \\
& D_{\mathbf{i}}^{\prime}=\left\{c \in D_{\mathbf{i}} \mid g_{c}^{k}\left(g_{c}(0)\right) \in U_{0, c}\right\},
\end{aligned}
$$

and for each $c \in D$ define

$4^{\mathrm{e}}$ SÉRIE - TOME $39-2006-\mathrm{N}^{\circ} 3$ 


$$
\begin{aligned}
U_{\mathbf{i}, c} & =\left\{z \in V_{c} \mid g_{c}^{j}(z) \in U_{i_{j}, c} \text { for } j=0,1, \ldots, k-1\right\} ; \\
W_{\mathbf{i}, c} & =\left\{z \in U_{\mathbf{i}, c} \mid g_{c}^{k}(z) \in U_{0, c}\right\} .
\end{aligned}
$$

Let, as before, $\mathcal{L} g_{c}$ denote the first return map of $g_{c}$ to the central domain $U_{0, c}$.

LEMMA 14. - For each $\mathbf{i}_{0}$, the renormalizations $\mathcal{L} g_{c}, c \in D_{\mathbf{i}_{0}}^{\prime}$ form an $R$-family.

Proof. - Let $\mathbf{h}: D \times \mathbb{C} \rightarrow D \times \mathbb{C}$ be an equipment for the family $\mathbf{g}:=\left\{g_{c}\right\}_{c \in D}$ so that $h_{c_{0}}=\operatorname{id}_{\mathbb{C}}$ for some $c_{0} \in D_{\mathbf{i}_{0}}^{\prime}$. Arguing as in the proof of Proposition 6 , we construct a holomorphic motion

$$
\Phi: D \times \mathbb{C} \rightarrow D \times \mathbb{C}, \quad(c, z) \mapsto\left(c, \varphi_{c}(z)\right)
$$

which is again an equipment of $\mathbf{g}$, and maps $W_{\mathbf{i}, c_{0}}$ onto $W_{\mathbf{i}, c}$. Next define a holomorphic motion $\widetilde{\Phi} \mid D_{\mathbf{i}_{0}}^{\prime} \times \mathbb{C}$ so that $\tilde{\varphi}_{c}(z)=\varphi(c, z)$ if $z \notin W_{\mathbf{i}_{0}}$ and $\tilde{\varphi}_{c}\left(c_{0}\right)=c$. By pulling back this $\widetilde{\Phi}$, we obtain a holomorphic motion which equips $\mathcal{L} g_{c}, c \in D_{\mathbf{i}_{0}}^{\prime}$ to an R-family.

For an R-family as in (11) we define

$$
\bmod (\mathbf{g})=\inf _{c \in D} \bmod \left(g_{c}\right)=\inf _{c \in D} \bmod \left(V_{c} \backslash \overline{U_{0, c}}\right),
$$

and

$$
\bmod ^{\prime}(\mathbf{g})=\inf _{c \in D} \bmod ^{\prime}\left(g_{c}\right)=\inf _{c \in D} \min \left\{\bmod \left(V_{c} \backslash \overline{U_{i, c}}\right): i\right\} .
$$

LEMMA 15. - Assume that $\bmod (\mathbf{g})$ is sufficiently large and that $\mathrm{g}$ is a well-controlled $R$-family. Then for each $\mathbf{i}_{0}$,

$$
\bmod \left(D_{\mathbf{i}_{0}} \backslash \bar{D}_{\mathbf{i}_{0}}^{\prime}\right) \geqslant \frac{1}{6} \bmod (\mathbf{g})-M,
$$

where $M>0$ is a universal constant. Moreover, $\mathcal{L} \mathrm{g}=\left\{\mathcal{L} g_{c}, c \in D_{\mathbf{i}_{0}}^{\prime}\right\}$ is again a well-controlled R-family.

Proof. - Let $\mathbf{h}$ and $\Phi$ be as in the proof of the previous lemma. Let $k=\left|\mathbf{i}_{0}\right|$. Note that the holomorphic motion $\Phi(c, z)=\left(c, \varphi_{c}(z)\right)$ constructed above maps $U_{\mathbf{i}_{0}, c_{0}}$ to $U_{\mathbf{i}_{0}, c}$ and $W_{\mathbf{i}_{0}, c_{0}}$ to $W_{\mathbf{i}_{0}}$. Since $\mathbf{g}$ is well-controlled, there exists a qc map $\psi_{c}$ which agrees with $h_{c}$ on $\partial V_{c_{0}} \cup\left(\bigcup_{i} \partial U_{i, c_{0}}\right)$ (and so is a pseudo-conjugacy between $g_{c_{0}}$ and $\left.g_{c}\right)$ and which is 2-qc outside $U_{0, c_{0}}$. Pulling back $\psi_{c}$ as before, we obtain for each $c \in D$, a qc map $\tilde{\varphi}_{c}: \mathbb{C} \rightarrow \mathbb{C}$ which coincides with $\varphi_{c}$ on $\partial U_{\mathbf{i}_{0}, c_{0}} \cup \partial W_{\mathbf{i}_{0}, c_{0}}$ and which is 2-qc outside $U_{0, c_{0}} \cup W_{\mathbf{i}_{0}, c_{0}}$. Since $\tilde{\varphi}_{c}$ is 2-qc only outside this set, we cannot apply Lemma 9 directly to this holomorphic motion. In order to obtain the estimate (12), in the following, we shall restrict the holomorphic motion to a smaller region $\widehat{D}_{\mathbf{i}_{0}}$.

Let $m:=\bmod \left(U_{\mathbf{i}_{0}, c_{0}} \backslash \overline{W_{\mathbf{i}_{0}, c_{0}}}\right)=\bmod \left(g_{c_{0}}\right)$. Let $\gamma \subset U_{\mathbf{i}_{0}, c_{0}} \backslash \overline{W_{\mathbf{i}_{0}, c_{0}}}$ be a Jordan curve which separates the annulus into two annuli with moduli equal to $m / 2$, and let $\widehat{D}=\widehat{D}_{\mathbf{i}_{0}}=$ $\left\{c \in D: g_{c}(0) \in h_{c}(\Omega)\right\}$, where $\Omega$ denotes the Jordan disk bounded by $\gamma$.

Let us prove that for each $c \in \widehat{D}$, there exists a 3 -qc map $\hat{\varphi}_{c}: \mathbb{C} \rightarrow \mathbb{C}$ which coincides with $\varphi_{c}$ on $\partial U_{\mathbf{i}_{0}, c_{0}} \cup \partial W_{\mathbf{i}_{0}, c_{0}}$. To this end it suffices to show that there exists a 3-qc map $\hat{\varphi}_{c}: U_{0, c_{0}} \rightarrow U_{0, c}$ which coincides with $h_{c}$ on $\partial U_{0, c_{0}}$. For each $c \in \widehat{D}$, the hyperbolic distance between $g_{c_{0}}(0), h_{c}^{-1}\left(g_{c}(0)\right) \in V_{c_{0}}$ is small (taking the hyperbolic metric on $V_{c_{0}}$ and assuming that $m$ is taken large enough). Thus there is a 3/2-qc map $\theta_{c}: V_{c_{0}} \rightarrow V_{c_{0}}$ which is equal to the identity on $\partial V_{c_{0}}$ and which maps $g_{c_{0}}(0)$ to $h_{c}^{-1}\left(g_{c}(0)\right)$. Thus the map $\hat{\psi}_{c}=h_{c} \circ \theta_{c}: V_{c_{0}} \rightarrow V_{c}$ is a 3-qc map which coincides with $h_{c}$ on $\partial V_{c_{0}}$ and satisfies $\psi_{c}\left(g_{c_{0}}(0)\right)=g_{c}(0)$. The statement follows. 
Now applying Lemma 9 to the holomorphic motion $\left\{\varphi_{c}: U_{\mathbf{i}_{0}, c_{0}} \rightarrow \mathbb{C}\right\}_{c \in \widehat{D}}$, we obtain that

$$
\bmod \left(\widehat{D} \backslash \overline{D_{\mathbf{i}_{0}}^{\prime}}\right) \geqslant m / 6-M,
$$

from which the estimate (12) follows.

When $\bmod (\mathbf{g})$ is sufficiently large, $\bmod \left(D \backslash D_{\mathbf{i}_{0}}^{\prime}\right) \geqslant \bmod \left(D_{\mathbf{i}_{0}} \backslash D_{\mathbf{i}_{0}}^{\prime}\right)$ is large, so by the optimal $\lambda$-lemma, $\varphi_{c}$ is 2-qc for all $c \in D_{\mathbf{i}_{0}}^{\prime}$. Therefore $\tilde{\varphi}_{c}$ is 2-qc outside $W_{\mathbf{i}_{0}, c_{0}}$. As an equipment of $\mathcal{L} \mathrm{g}$ is obtained by pull back the holomorphic motion $\widetilde{\Phi}$, it follows that $\mathcal{L} \mathrm{g}$ is well-controlled.

Remark 8. - It is clear from the argument above that if $\mathbf{g}$ is a standard R-family, then for any $\mathbf{i}$, $D_{\mathbf{i}}, D_{\mathbf{i}}^{\prime}$ are parapuzzle pieces, and the family $\mathcal{L} \mathrm{g}$ is again a standard family.

Before stating the next proposition, let us first give a fact on the capacity.

LEMMA 16. - Let $\Omega \ni \Omega^{\prime}$ be real-symmetric Jordan disks, and let $J \supset J^{\prime}$ be their real traces. Assume that $\bmod \left(\Omega \backslash \overline{\Omega^{\prime}}\right)$ is sufficiently large. Then for each $\gamma \geqslant 1$ there exists $\eta=\eta(\gamma)$ such that

$$
\operatorname{Cap}_{\gamma}\left(J^{\prime}, J\right) \leqslant \exp \left(-\eta \bmod \left(\Omega \backslash \overline{\Omega^{\prime}}\right)\right) .
$$

Proof. - It is well-known that provided that $\bmod \left(\Omega \backslash \overline{\Omega^{\prime}}\right)$ is large enough, for any $z_{0} \in \Omega^{\prime}$ there exists a round annulus $A=\left\{r<\left|z-z_{0}\right|<R\right\} \subset \Omega \backslash \overline{\Omega^{\prime}}$ with $\bmod (A) \geqslant$ $\bmod \left(\Omega \backslash \overline{\Omega^{\prime}}\right)-M$, where $M$ is a universal constant. Let us take $z_{0} \in \Omega^{\prime} \cap \mathbb{R}$, $T=\left(z_{0}-R, z_{0}+R\right), T^{\prime}=\left(z_{0}-r, z_{0}+r\right)$. Then clearly, $J^{\prime} \subset T^{\prime} \subset T \subset J$, so $\operatorname{Cap}_{\gamma}\left(J^{\prime}, J\right) \leqslant$ $\operatorname{Cap}_{\gamma}\left(T^{\prime}, T\right)$. For each $\gamma$-quasisymmetric map $h$ from $T$ into $\mathbb{R}$, clearly $\left|h T^{\prime}\right| /|h T|$ is bounded from above by a power of $r / R$. The lemma follows.

Proposition 7. - Let $c_{0} \in \mathcal{F}^{0}$ and let $\mathrm{g}$ be a standard $R$-family over $D=\mathcal{P}_{m}\left(c_{0}\right)$. Assume that the R-family $\mathbf{g}=\left\{g_{c}\right\}$ is well-controlled, and that $\bmod (\mathbf{g})$ is sufficiently large. Then for any $\gamma \geqslant 1$ there exists $\eta>0$ such that

$$
\operatorname{Cap}_{\gamma}(\widetilde{D} \cap \mathbb{R}, D \cap \mathbb{R}) \leqslant \exp (-\eta \bmod (\mathbf{g})), \quad \text { where } \widetilde{D}=\bigcup_{|\mathbf{i}| \leqslant 4 \ell^{2}} D_{\mathbf{i}}^{\prime} .
$$

Proof. - Let $J_{\mathbf{i}}=D_{\mathbf{i}} \cap \mathbb{R}$ and $J_{\mathbf{i}}^{\prime}=D_{\mathbf{i}}^{\prime} \cap \mathbb{R}$. By Lemma 15, provided that $\bmod (\mathbf{g})$ is large enough, for any word $\mathbf{i}$ we have

$$
\bmod \left(D_{\mathbf{i}} \backslash \bar{D}_{\mathbf{i}}^{\prime}\right) \geqslant \bmod (\mathbf{g}) / 6-M>\bmod (\mathbf{g}) / 7
$$

By Lemma 16, this implies that

$$
\operatorname{Cap}_{\gamma}\left(J_{\mathbf{i}}^{\prime}, J_{\mathbf{i}}\right) \leqslant \exp \left(-\frac{\eta}{7} \bmod (\mathbf{g})\right) .
$$

For any $k \geqslant 0$, the $J_{\mathbf{i}}$ 's with $|\mathbf{i}|=k$ are pairwise disjoint, thus

$$
\operatorname{Cap}_{\gamma}\left(\bigcup_{|\mathbf{i}|=k} J_{\mathbf{i}}^{\prime}, D \cap \mathbb{R}\right) \leqslant \sup _{|\mathbf{i}|=k} \operatorname{Cap}_{\gamma}\left(J_{\mathbf{i}}^{\prime}, J_{\mathbf{i}}\right) \leqslant \exp \left(-\frac{\eta}{7} \bmod (\mathbf{g})\right) .
$$

$4^{\text {e }}$ SÉRIE - TOME $39-2006-\mathrm{N}^{\circ} 3$ 
Therefore

$$
\operatorname{Cap}_{\gamma}(\widetilde{D} \cap \mathbb{R}, D \cap \mathbb{R}) \leqslant\left(4 \ell^{2}+1\right) \exp \left(-\frac{\eta}{7} \bmod (\mathbf{g})\right) .
$$

Redefining the constant $\eta$ completes the proof.

\subsection{Proof of Theorem 4}

The proof of Theorem 4 is based on the following lemmas.

Lemma 17. - Let $c_{0} \in \mathcal{D G}$. Then for any $C>0$ there exists a standard $R$-family $\mathbf{g}$ over some parapuzzle piece $\mathcal{P}_{m}\left(c_{0}\right)$ such that $\mathbf{g}$ is well-controlled and

$$
\bmod (\mathbf{g}) \geqslant 2 \ell C, \quad \bmod ^{\prime}(\mathbf{g}) \geqslant C .
$$

Proof. - Let $\varepsilon>0$ be a small number. By Theorem 6, there exists an arbitrarily large $n \in \mathbb{N}$ such that the domain of the first return map to $P_{c_{0}}^{n}(0)$ under $f_{c_{0}}$ is $\varepsilon$-absolutely small in $P_{c_{0}}^{n}(0)$. By Proposition 6, there is a parapuzzle piece $\mathcal{P}_{m^{\prime}}\left(c_{0}\right)$ such that $\hat{\mathrm{g}}=\left\{g_{c}\right\}_{c \in \mathcal{P}_{m^{\prime}}\left(c_{0}\right)}$ forms a standard $R$-family, where $g_{c}$ denotes the first return map to $P_{c}^{n}(0)$ under $f_{c}$. Provided that $\varepsilon$ was chosen sufficiently small, by Proposition 5 and the last statement of Proposition 6, this family is well-controlled and thus $\bmod (\hat{\mathbf{g}}) \geqslant \bmod \left(g_{c_{0}}\right) / 2$ is large. By Lemma 15, there is a smaller parapuzzle piece $\mathcal{P}_{m}\left(c_{0}\right)$ (with $m>m^{\prime}$ ) such that $\mathcal{L} g_{c}, c \in \mathcal{P}_{m}\left(c_{0}\right)$ forms another standard wellcontrolled $R$-family g. Moreover, by Lemma $8, \bmod (\mathbf{g})$ and $\bmod ^{\prime}(\mathbf{g})$ are both large.

Recall that $\mathcal{S C}$ is the subset of $\mathcal{F}^{0}$ consisting of all the parameters $c$ for which the summability condition (1) holds for all $\alpha>0$. In the following we shall use the following criterion:

LEMMA 18. - Let $c \in \mathcal{F}^{0}$. Then $c \in \mathcal{S C}$ if one of the following holds:

(1) $c \notin \mathcal{F}_{r}^{0}$;

(2) for $f_{c}$, there exists a nice interval $I \ni 0$ with the following property: if we define $I^{0}=I$ and define $I^{k+1}$ to be the central return domain to $I^{k}$, then $\left|I^{i+1}\right| /\left|I^{i}\right|$ decreases to 0 at least exponentially fast.

Proof. - In the first case, the map has no periodic attractor and the critical point is nonrecurrent. By a well-known theorem of Mañé, see for example Section III.5 in [29], this implies that $f_{c}$ satisfies the Collet-Eckmann condition: $\left|D f_{c}^{n}(c)\right|$ is exponentially big in $n$, which implies that $c \in \mathcal{S C}$. In the second case, the result was proved in [28].

LEMMA 19. - For any $\delta>0$ and $\gamma \geqslant 1$, there exists $C>0$ with the following property. Let $\mathrm{g}$ be a well-controlled standard $R$-family over a parapuzzle $\mathcal{P}_{m}\left(c_{0}\right)$ with $c_{0} \in \mathbb{R}$ such that $\bmod (\mathbf{g}) \geqslant 2 \ell C$ and $\bmod ^{\prime}(\mathbf{g}) \geqslant C$. Let $T=\mathcal{P}_{m}\left(c_{0}\right) \cap \mathbb{R}$. Then

$$
\operatorname{Cap}_{\gamma}(T \backslash \mathcal{S C}, T) \leqslant \delta
$$

Proof. - The strategy is to construct a sequence of open sets

$$
\Omega^{(0)}=\mathcal{P}_{m}\left(c_{0}\right) \supset \Omega^{(1)} \supset \Omega^{(2)} \supset \cdots
$$

with the following properties:

- for each $k, \Omega^{(k)}$ is a disjoint union of parapuzzle pieces $\Omega^{(k, j)}$ which intersect $\mathbb{R}$ and defined by the renormalization procedure described in Section 7.2; 
- for each $(k, j)$, there exists a standard R-family $\tilde{\mathbf{g}}=\mathbf{g}^{(k, j)}$ over $\Omega^{(k, j)}$ which is wellcontrolled and

$$
\bmod (\tilde{\mathbf{g}}) \geqslant 2^{k+1} \ell C, \quad \bmod ^{\prime}(\tilde{\mathbf{g}}) \geqslant 2^{k} C ;
$$

- for each component $\mathcal{P}$ of $\Omega^{(k)}$, we have

$$
\operatorname{Cap}_{\gamma}\left((\mathcal{P} \cap \mathbb{R}) \backslash\left(\Omega^{(k+1)} \cup \mathcal{S C}\right), \mathcal{P} \cap \mathbb{R}\right) \leqslant 2^{-k-1} \delta .
$$

The existence of these $\Omega^{(k)}$ completes the proof. In fact, Eq. (14) implies that

$$
\operatorname{Cap}_{\gamma}\left(\left(T \backslash \bigcap_{k} \Omega^{(k)}\right) \backslash \mathcal{S C}, T\right) \leqslant \delta .
$$

Moreover, by Lemma 18, the modulus estimate (13) shows that for any $c \in T \cap \bigcap_{k} \Omega^{(k)}, c \in \mathcal{S C}$.

Let us construct these sets by induction. The choice of $\Omega^{(0)}$ satisfies the requirement by assumption. Assume now that $\Omega^{(k)}$ is constructed. Take a component $\mathcal{P}$ of $\Omega^{(k)}$, and let $\hat{\mathrm{g}}$ be the $\mathrm{R}$-family over $\mathcal{P}$ which is given by the induction assumption. For each word $\mathbf{i}$ of positive integers, define $\mathcal{P}_{\mathbf{i}}$ and $\mathcal{P}_{\mathrm{i}}^{\prime}$ as in the previous subsection. The set $\Omega^{(k+1)}$ is defined to be the union of all sets of the form $\mathcal{P}_{\mathbf{i}}^{\prime}$ with $|\mathbf{i}|>4 \ell^{2}$ which intersect $\mathbb{R}$. This is clearly a disjoint union of parapuzzle pieces intersecting $\mathbb{R}$. By Lemma 15 , for each $\mathcal{P}_{\mathbf{i}}, \mathcal{L} \hat{g}_{c}, c \in \mathcal{P}_{\mathbf{i}}$ form a well-controlled R-family. Applying Lemma 8 to $\hat{g}_{c}$, we obtain

$$
\begin{aligned}
\bmod \left(\mathcal{L} \hat{g}_{c}\right) & \geqslant \frac{(|\mathbf{i}|-1) \bmod ^{\prime}\left(\hat{g}_{c}\right)+\bmod \left(\hat{g}_{c}\right)}{\ell} \\
& \geqslant \frac{\left(4 \ell^{2}-1\right) \bmod ^{\prime}\left(\hat{g}_{c}\right)+\bmod \left(\hat{g}_{c}\right)}{\ell} \geqslant 2^{k+2} \ell C,
\end{aligned}
$$

and

$$
\bmod ^{\prime}\left(\mathcal{L} \hat{g}_{c}\right) \geqslant \frac{\bmod \left(\hat{g}_{c}\right)}{\ell} \geqslant 2^{k+1} C .
$$

By Proposition 7, for each component $\mathcal{P}$ of $\Omega^{(k)}$,

$$
\begin{aligned}
\operatorname{Cap}_{\gamma}\left(\bigcup_{|\mathbf{i}| \leqslant 4 \ell} \mathcal{P}_{\mathbf{i}} \cap \mathbb{R}, \mathcal{P} \cap \mathbb{R}\right) & \leqslant \exp (-\eta \bmod (\hat{\mathbf{g}})) \\
& \leqslant \exp \left(-2^{k} \ell \eta C\right) \leqslant 2^{-k-1} \delta,
\end{aligned}
$$

provided that $C$ is sufficiently large. Note that $\left(\mathcal{P} \backslash \bigcup_{\mathbf{i}} \mathcal{P}_{\mathbf{i}}\right) \cap \mathbb{R} \subset \mathcal{F}^{0} \backslash \mathcal{F}_{r}^{0}$, so by Lemma 18,

$$
\mathcal{P} \backslash\left(\Omega^{(k+1)} \cup \mathcal{S C}\right) \subset \bigcup_{|\mathbf{i}| \leqslant 4 \ell^{2}} \mathcal{P}_{\mathbf{i}}
$$

This completes the construction and thus the proof of the lemma.

We finish with the

Proof of Theorem 4. - Combining Lemmas 17 and 19, we obtain the theorem.

\section{Acknowledgement}

We are indebted to the referee for his/her comments on and very careful reading of previous versions of this paper.

$4^{e}$ SÉRIE - TOME $39-2006-\mathrm{N}^{\circ} 3$ 


\section{REFERENCES}

[1] Ahlfors L., Lectures on Quasiconformal Mappings, Van Nostrand, Princeton, NJ, 1966.

[2] Avila A., Lyubich M., De Melo W., Regular or stochastic dynamics in real analytic families of unimodal maps, Invent. Math. 154 (2003) 451-550.

[3] Avila A., Moreira C.G., Statistical properties of unimodal maps: The quadratic family, Ann. of Math. 161 (2005) 831-881.

[4] Birkhoff G., Extensions of Jentzsch's theorem, Trans. Amer. Math. Soc. 85 (1957) 219-227.

[5] Blokн A., Lyubich M., Measurable dynamics of S-unimodal maps of the interval, Ann. Sci. École Norm. Sup. 24 (1991) 737-749.

[6] Bruin H., Minimal Cantor systems and unimodal maps, J. Difference Eq. Appl. 9 (2003) 305-318.

[7] BRUIN H., Topological conditions for the existence of Cantor attractors, Trans. Amer. Math. Soc. 350 (1998) 2229-2263.

[8] Bruin H., LuzZatto S., van Strien S., Decay of correlations in one-dimensional dynamics, Ann. Sci. École Norm. Sup. 36 (2003) 621-646.

[9] Bruin H., Keller G., Nowicki T., van Strien S., Wild Cantor attractors exist, Ann. of Math. 143 (1996) 97-130.

[10] BRUIN H., SHEN W., VAN STRIEN S., Invariant measures exist without a growth condition, Commun. Math. Phys. 241 (2-3) (2003) 287-306.

[11] Douady A., Hubbard J., Dynamical study of complex polynomials, Part I and Part II, Mathematical Publications of Orsay 84-2, 85-4 (in French).

[12] Doundy A., HubBard J., On the dynamics of polynomial-like mappings, Ann. Sci. École Norm. Sup. (4) 18 (2) (1985) 287-343.

[13] DURAND F., Linearly recurrent subshifts have a finite number of non-periodic subshift factors, Ergodic Theory Dynam. Syst. 20 (2000) 1061-1078.

[14] Graczyk J., Świştek G., Induced expansion for quadratic polynomials, Ann. Sci École Norm. Súp. 29 (1996) 399-482.

[15] Hasselblatt B., Katok A., A First Course in Dynamics, with a Panorama of Recent Developments, Cambridge University Press, Cambridge, 2003.

[16] Hofbauer F., Keller G., Some remarks on recent results about S-unimodal maps, Ann. Inst. Henri Poincaré 53 (1990) 413-425.

[17] KAHN J., Holomorphic removability of Julia sets, IMS preprint ims $98-11$.

[18] KoZLOVSKi O., Getting rid of the negative Schwarzian derivative condition, Ann. of Math. 152 (2000) 743-762.

[19] Kozlovski O., Shen W., VAn Strien S., Rigidity for real polynomials, Ann. of Math., in press.

[20] Kozlovski O., Shen W., van Strien S., Density of Axiom A in dimension one, Ann. of Math., in press.

[21] LYUBICH M., Combinatorics, geometry and attractors of quasi-quadratic maps, Ann. of Math. 140 (1994) 347-404 and Erratum Manuscript (2000).

[22] LYUBICH M., Dynamics of quadratic polynomials. III. Parapuzzle and SRB measures, in: Géométrie complexe et systèmes dynamiques, Orsay, 1995, in: Astérisque, vol. 261, 2000, pp. xii-xiii, 173200.

[23] LyUbich M., Feigenbaum-Coullet-Tresser universality and Milnor's hairiness conjecture, Ann. of Math. 149 (2) (1999) 319-420.

[24] LYUBICH M., Almost every real quadratic map is either regular or stochastic, Ann. of Math. 156 (2002) $1-78$.

[25] McMullen C., Complex Dynamics and Renormalization, Ann. of Math Stud., vol. 135, 1994.

[26] MAÑé R., Ergodic Theory and Differentiable Dynamics, Springer, New York, 1987.

[27] Martens M., Distortion results and invariant Cantor sets of unimodal maps, Ergodic Theory Dynam. Syst. 14 (2) (1994) 331-349.

[28] Martens M., Nowicki T., Invariant measures for typical quadratic maps, in: Géométrie complexe et systèmes dynamiques, Orsay, 1995, in: Astérisque, vol. 261, 2000, pp. 239-252.

[29] De Melo W., VAn StRIEN S., One-Dimensional Dynamics, Springer, Berlin, 1993. 
[30] Milnor J., Periodic orbits, externals rays and the Mandelbrot set: An expository account, in: Géométrie complexe et systèmes dynamiques, Orsay, 1995, in: Astérisque, vol. 261, 2000, pp. xiii, 277-333.

[31] NOWICKI T., VAN STRIEN S., Invariant measures exist under a summability condition, Invent. Math. 105 (1991) 123-136.

[32] RiVERA-LETELIER J., Rational maps with decay of geometry: Rigidity, Thurston's algorithm and local connectivity, IMS preprint ims00-09.

[33] Roesch P., Holomorphic motions and puzzles (following M. Shishikura), in: The Mandelbrot set theme and variations, in: London Math. Soc. Lecture Note Ser., vol. 274, Cambridge University Press, Cambridge, 2000, pp. 117-131.

[34] SHEN W., Bounds for one-dimensional maps without inflection critical points, J. Math. Sci. Univ. Tokyo 10 (1) (2003) 41-88.

[35] SHEN W., Decay geometry for unimodal maps: an elementary proof, Ann. of Math. (2) 163 (2) (2006) 383-404.

[36] SHISHIKURA M., Yoccoz puzzles, $\tau$-functions and their applications, Unpublished.

[37] SLOdKOWski Z., Holomorphic motions and polynomial hulls, Proc. Amer. Math. Soc. 111 (2) (1991) 347-355.

[38] VAN STRIEN S., VARGAS E., Real bounds, ergodicity and negative Schwarzian for multimodal maps, J. Amer. Math. Soc. 17 (2004) 749-782.

[39] Yoccoz J.-C., Unpublished.

(Manuscrit recu le 29 avril 2004; accepté, après révision, le 21 février 2006.)

Henk BRUIN

Mathematics and Statistics, University of Surrey, Guildford, Surrey, GU2 7XH, UK

E-mail: h.bruin@surrey.ac.uk

http://www.maths.surrey.ac.uk/showstaff?H.Bruin

Weixiao SHEN

Department of Mathematics,

University of Sciences and Technology of China,

Hefei 230026, China PR

E-mail: wxshen@ustc.edu.cn

Sebastian VAN STRIEN

Department of Mathematics,

University of Warwick,

Coventry CV4 7AL, UK

E-mail: strien@maths.warwick.ac.uk

http://www.maths.warwick.ac.uk/ strien

4 SÉRIE - TOME $39-2006-\mathrm{N}^{\circ} 3$ 